\title{
Recursion relation for boundary contribution
}

\author{
Qingjun $\operatorname{Jin}^{a}$ and Bo Feng ${ }^{a, b, 1}$ \\ ${ }^{a}$ Zhejiang Institute of Modern Physics, Zhejiang University, \\ Hangzhou, 310027, P.R. China \\ ${ }^{b}$ Center of Mathematical Science, Zhejiang University, \\ Hangzhou, P.R. China \\ E-mail: qingjun@zju.edu.cn, b.feng@cms.zju.edu.cn
}

ABSTRACT: It is well known that under a BCFW-deformation, there is a boundary contribution when the amplitude scales as $\mathcal{O}\left(z^{0}\right)$ or worse. We show that boundary contributions have a similar recursion relation as scattering amplitude. Just like the BCFW recursion relation, where scattering amplitudes are expressed as the products of two on-shell subamplitudes (plus possible boundary contributions), our new recursion relation expresses boundary contributions as products of sub-amplitudes and boundary contributions with less legs, plus yet another possible boundary contribution. In other words, the complete scattering amplitude, including boundary contributions, can be obtained by multiple steps of recursions, unless the boundary contributions are still non-zero when all possible deformations are exploited. We demonstrate this algorithm by several examples. Especially, we show that for standard model like renormalizable theory in 4D, i.e., the theory including only gauge boson, fermions and scalars, the complete amplitude can always be computed by at most four recursive steps using our algorithm.

KEywords: Scattering Amplitudes, Effective field theories

ArXIV EPRINT: 1412.8170

\footnotetext{
${ }^{1}$ The unusual ordering of authors is just to let authors get proper recognition of contributions under outdated practice in China.
} 


\section{Contents}

1 The introduction 1

2 The recursion relation for boundary contribution $\quad 2$

2.1 Recursion relation for boundary contribution 3

3 Scalar theory 5

4 The standard model like theory $\quad 6$

4.1 The case (c) with only two fermions 8

$\begin{array}{lll}4.2 & \text { The case (d) with only scalars } & 9\end{array}$

5 Examples 13

5.1 Example I: two fermions with three scalars 13

$\begin{array}{lll}5.2 & \text { Example II: six scalars } & 14\end{array}$

$\begin{array}{llr}6 & \text { Discussions } & 16\end{array}$

$\begin{array}{ll}\text { A Ordering of integration and residues of multiple variables } & 17\end{array}$

$\begin{array}{lr}\text { B Other deformations for boundary } & 19\end{array}$

$\begin{array}{lll}\text { B.1 Using Risager's deformation } & 19\end{array}$

B.2 Using the deformation $\langle 2| 3] \quad 20$

$\begin{array}{ll}\text { C Light cone propagator } & 22\end{array}$

\section{The introduction}

In recent years $\mathrm{BCFW}$ recursion relation $[1,2]$ has become a standard method to compute tree-level scattering amplitudes. In its original form, BCFW recursion relation was presented for 4d Yang-Mills theory in the language of spinors, but soon the method were applied to various other theories. ${ }^{1}$ Despite its successes, BCFW recursion relation has met difficulties applying to certain theories ${ }^{2}$ whose amplitudes do not have the desired vanishing scaling in the large limit of deformation parameter. A naive application of BCFW recursion relation fails to capture a piece of amplitude (usually called boundary contributions), which corresponds to the residue at infinity.

Several proposals have been made to find boundary contributions. The first $[6,7]$ is to introduce auxiliary fields so that in the enlarged theory, there are no boundary contributions. The second [8-10] is to carefully analyze Feynman diagrams and then isolate their

\footnotetext{
${ }^{1}$ For more information, see reviews [3-5] and references therein.

${ }^{2} \mathrm{~A}$ typical example is when all external particles are scalars and fermions in the Standard Model.
} 
boundary contributions, which can be evaluated directly or recursively afterwards. The third [11-13] is to express boundary contributions in terms of roots of amplitudes. These three methods are, however, effective only for limited types of theories. Recently a systematical algorithm, based on carefully analysis of pole structure of boundary contributions, has been proposed in [14]. Though in principle the method is applicable to any quantum field theory, in practice it suffers from high computational complexity.

In this paper, we present a new method to compute boundary contributions. The key observation is that with properly chosen deformations, boundary contributions satisfy similar recursion relations as scattering amplitudes. Just like the BCFW recursion relation, where scattering amplitudes are expressed as (a sum of) the products of two on-shell sub-amplitudes (plus possible boundary contribution), our new recursion relation expresses boundary contributions as (a sum of) products of sub-amplitudes and boundary contributions with less legs, plus yet another possible boundary contribution. The new boundary contribution is subsequently computed by a new shift, and the recursion ends whenever the remaining boundary contribution vanishes. This multi-step recursion is (almost) as efficient as BCFW recursion, but applicable to more general models.

The paper is organized as follows. In section 2 after a short discussion of pole structure, we present our main result: the recursion relations for boundary contributions. In section 3 we show that a pure scalar $\phi^{m}$ theory amplitude can be computed via a $(m-1)$ step recursion. In section 4 we analyze all possible boundary contributions of Standard Model like theories, and show that any amplitude in this theory can be computed via a (at most) 4 step recursion. In section 5, we present two explicit examples using our method. In appendix A, we discuss some mathematical aspects of multi-variable integrations. In appendix B, we present recursion relation for boundary contributions under other choices of deformations. In appendix C, propagator in light-cone gauge has been discussed.

\section{The recursion relation for boundary contribution}

The key idea of BCFW recursion is determining scattering amplitudes by their poles. In order to find a recursion relation of boundary contributions, we also need to be very clear about the poles of the boundary contribution. First consider the primary deformation (BCFW-deformation) $\langle 1| n]$,

$$
\lambda_{1} \rightarrow \lambda_{1}-z \lambda_{n}, \quad \widetilde{\lambda}_{n} \rightarrow \widetilde{\lambda}_{n}+z \widetilde{\lambda}_{1}
$$

Let us use indices $I, J$ to denote subsets of remaining particles $\mathcal{T} \equiv\{2,3, \ldots, n-1\}$. For later convenience, we also define $q_{i}^{\mu}=\frac{1}{2}\left[i\left|\gamma^{\mu}\right| n\right\rangle$, then (2.1) can be written as

$$
p_{1} \rightarrow p_{1}-z q_{1}, p_{n} \rightarrow p_{n}+z q_{1} \text {. }
$$

Under the deformation, the expression of tree-level amplitudes coming from Feynman diagrams will be

$$
A(z)=\frac{f(z)}{\prod_{I \subset \mathcal{T}} P_{I}^{2} \prod_{J \subset \mathcal{T}}\left(P_{J}+p_{1}-z q_{1}\right)^{2}}
$$




$$
=\sum_{J \subset \mathcal{T}} \frac{R_{J}}{\left(P_{J}+p_{1}-z q_{1}\right)^{2}}+B^{\langle 1| n]}+C_{1} z+C_{2} z^{2}+\cdots
$$

where $R_{J}$ 's are the residues of corresponding poles and $B^{\langle 1| n]}$ is the boundary contribution we want to find.

To read out $B^{\langle 1| n]}$, a good way is to do the large $z$ expansion in the first line of (2.3). Using

$$
\frac{1}{\left(P_{J}+p_{1}-z q_{1}\right)^{2}}=\frac{1}{-z\left\langle n\left|P_{J}+p_{1}\right| 1\right]} \sum_{i=0}^{\infty}\left(\frac{\left(P_{J}+p_{1}\right)^{2}}{z\left\langle n\left|P_{J}+p_{1}\right| 1\right]}\right)^{i}
$$

we found that

$$
A(z)=\frac{f(z)}{\prod_{I \subset \mathcal{T}} P_{I}^{2}} \prod_{J \subset \mathcal{T}}\left[\frac{1}{-z\left\langle n\left|P_{J}+p_{1}\right| 1\right]} \sum_{i=0}^{\infty}\left(\frac{\left(P_{J}+p_{1}\right)^{2}}{z\left\langle n\left|P_{J}+p_{1}\right| 1\right]}\right)^{i}\right]
$$

and $B^{\langle 1| n]}$ can be read out by selecting same power of $z$ in numerator $f(z)$ and denominators. In other words, poles of $B$ can be

$$
P_{I \subset \mathcal{T}}^{2}, \quad\left\langle n\left|P_{J \subset \mathcal{T}}\right| 1\right]^{a}
$$

It is worth to notice that, in principle, $B^{\langle 1| n]}$ can have terms which are pure polynomials in momentum (i.e. they do not have any pole), and our method is not applicable. This can happen in many effective theories with higher dimension operators.

\subsection{Recursion relation for boundary contribution}

As discussed in [14], we can use a different deformation to compute $B^{\langle 1| n]}$. Without loss of generality, we will choose the deformation $\langle 2| n]$. A crucial merit of this deformation is that spurious poles $\left\langle n\left|P_{J \subset \mathcal{T}}\right| 1\right]$ in (2.6) as well as others $\left\langle n\left|P_{J \subset \mathcal{T}}\right| i\right]$ generated in middle steps are invariant under the deformation. In other words, under this deformation only physical single poles $P_{I \subset \mathcal{T}}^{2}$ in $(2.6)$ are detected.

Following the proof of $\mathrm{BCFW}$ recursion relations, we evaluate the contour integration

$$
B^{\langle 12| n]}=\frac{1}{2 \pi i} \oint_{|w|=R} d w \frac{B^{\langle 1| n]}(w)}{w}=B^{\langle 1| n]}+\sum_{w^{*}} \operatorname{Res}\left(\frac{B^{\langle 1| n]}(w)}{w}\right)_{w=w^{*}} .
$$

where $B^{\langle 12| n]}$ is the possible remaining boundary contribution and the residue part is given by recursion relation

$$
-\operatorname{Res}\left(\frac{B^{\langle 1| n]}}{w}\right)_{w=w_{\mathcal{I}}}=\sum_{h} A_{L}\left(\widehat{p}_{2}\left(w_{\mathcal{I}}\right), \mathcal{I},-P^{h}\left(w_{\mathcal{I}}\right)\right) \frac{1}{\left(p_{2}+P_{\mathcal{I}}\right)^{2}} B^{\langle 1| n]}\left(p_{1}, \widehat{p}_{n}\left(w_{\mathcal{I}}\right), \overline{\mathcal{I}}, P^{-h}\left(w_{\mathcal{I}}\right)\right)
$$

with $w_{\mathcal{I}}=\frac{\left(p_{2}+P_{\mathcal{I}}\right)^{2}}{\left\langle n\left|P_{\mathcal{I}}\right| 2\right|}$ and $^{3} \quad \mathcal{I} \cup \overline{\mathcal{I}}=\{3,4, \ldots, n-1\} . \quad$ In $(2.8)$, the $B^{[1|n\rangle}\left(p_{1}, \widehat{p}_{n}\left(w_{\mathcal{I}}\right), \overline{\mathcal{I}}, P^{-h}\left(w_{\mathcal{I}}\right)\right)$ is the boundary contribution of lower point amplitudes under deformation $\langle 1| n]$.

\footnotetext{
${ }^{3}$ Here $1 \notin \mathcal{I} \bigcup \overline{\mathcal{I}}$, because according to $(2.6), B^{\langle 1| n]}$ does not have poles at $\left(p_{1}+p_{2}+P_{J}\right)^{2}$.
} 


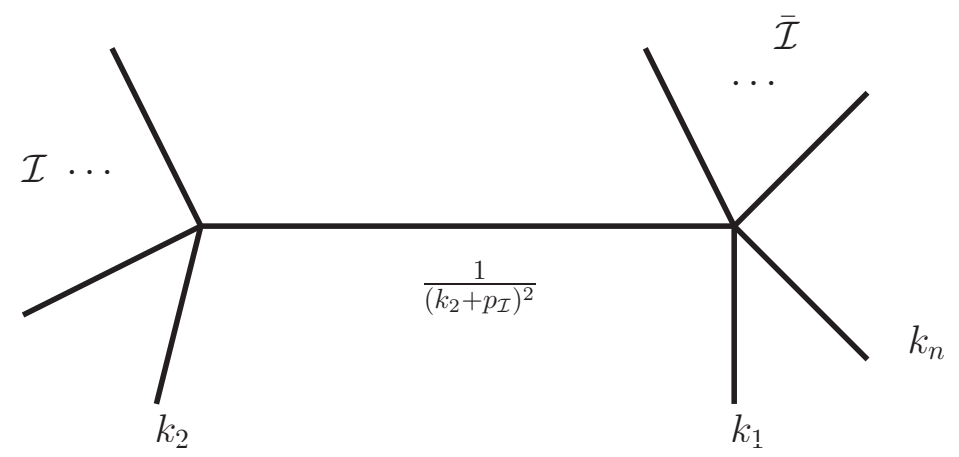

Figure 1. Recursion relation of $B^{\langle 1| n]}$ using the $\left.\langle 2| n\right]$ deformation. The left hand side of the diagram is an on-shell sub-amplitude, while the right hand side of the diagram is the boundary $B^{\langle 1| n]}$ with less legs.

To prove (2.8), first we notice that

$$
B^{\langle 1| n]}\left(p_{1}, p_{2}, \ldots, p_{n-1}, p_{n}\right)=\frac{1}{2 \pi i} \oint_{|z|=R} \frac{d z}{z} A\left(p_{1}-z q_{1}, p_{2}, \ldots, p_{n-1}, p_{n}+z q_{1}\right)
$$

thus

$$
\begin{aligned}
& -\operatorname{Res}\left(\frac{B^{\langle 1| n]}}{w}\right)_{w=w_{\mathcal{I}}}=-\frac{1}{2 \pi i} \oint_{w=w_{\mathcal{I}}} \frac{d w}{w} B^{\langle 1| n]}\left(p_{1}, p_{2}-w q_{2}, \ldots, p_{n-1}, p_{n}+w q_{2}\right) \\
& =-\frac{1}{(2 \pi i)^{2}} \oint_{w=w_{\mathcal{I}}} \frac{d w}{w} \oint_{|z|=R} \frac{d z}{z} A\left(p_{1}-z q_{1}, p_{2}-w q_{2}, p_{3}, \ldots, p_{n-1}, p_{1}+z q_{1}+w q_{2}\right)
\end{aligned}
$$

where we have used (2.9) at the second line. Above integration can be parameterized by $w=w_{\mathcal{I}}+\epsilon e^{i \alpha}$ and $z=R e^{i \beta}$, thus the contour integration becomes following double integrations

$$
-\frac{\epsilon}{(2 \pi)^{2}} \int_{0}^{2 \pi} \frac{d \alpha d \beta}{w_{\mathcal{I}}+\epsilon e^{i \alpha}} A\left(p_{1}-R e^{i \beta} q_{1}, p_{2}-\left(w_{\mathcal{I}}+\epsilon e^{i \alpha}\right) q_{2}, p_{3}, \ldots, p_{n-1}, p_{1}+R e^{i \beta} q_{1}+\left(w_{\mathcal{I}}+\epsilon e^{i \alpha}\right) q_{2}\right)
$$

Now for $R$ big enough but finite and $\epsilon$ small enough but finite, $A$ is finite (i.e., there is no pole along the integral path). Using the Fubini-Tonelli theorem reviewed in appendix A, we can exchange the ordering of two integrations, thus (2.10) becomes

$$
\begin{aligned}
& -\frac{1}{(2 \pi i)^{2}} \oint_{|z|=R} \frac{d z}{z} \oint_{w=w_{\mathcal{I}}} \frac{d w}{w} A\left(p_{1}-z q_{1}, p_{2}-w q_{2}, p_{3}, \ldots, p_{n-1}, p_{1}+z q_{1}+w q_{2}\right) \\
& =\frac{1}{2 \pi i} \sum_{h} A_{L}\left(\widehat{p}_{2}\left(w_{\mathcal{I}}\right), \mathcal{I},-P^{h}\left(w_{\mathcal{I}}\right)\right) \frac{1}{\left(p_{2}+P_{\mathcal{I}}\right)^{2}} \oint_{|z|=R} \frac{d z}{z} A_{R}\left(p_{1}-z q_{1}, \overline{\mathcal{I}}, p_{n}+z q_{1}+w_{\mathcal{I}} q_{2}, P^{-h}\left(w_{\mathcal{I}}\right)\right) \\
& =\sum_{h} A_{L}\left(\widehat{p}_{2}\left(w_{\mathcal{I}}\right), \mathcal{I},-P^{h}\left(w_{\mathcal{I}}\right)\right) \frac{1}{\left(p_{2}+P_{\mathcal{I}}\right)^{2}} B^{\langle 1| n]}\left(p_{1}, \widehat{p}_{n}\left(w_{\mathcal{I}}\right), \overline{\mathcal{I}}, P^{-h}\left(w_{\mathcal{I}}\right)\right)
\end{aligned}
$$

Thus we have proved (2.8). If $B^{\langle 12| n]} \neq 0$, we can take a third deformation, for example $\langle 3| n]$. First we write

$$
B^{\langle 12| n]}=\frac{1}{(2 \pi i)^{2}} \oint_{\left|z_{2}\right|=R_{2}} \frac{d z_{2}}{z_{2}} \oint_{\left|z_{1}\right|=R_{1}} \frac{d z_{1}}{z_{1}} A\left(p_{1}-z_{1} q_{1}, p_{2}-z_{2} q_{2}, \cdots, p_{n}+z_{1} q_{1}+z_{2} q_{2}\right)
$$


Using the contour integration, we obtain

$$
B^{\langle 12| n]}=B^{\langle 123| n]}-\sum_{w_{\mathcal{I}}} \operatorname{Res}\left(\frac{B^{\langle 12| n]}(w)}{w}\right)_{w_{\mathcal{I}}}
$$

It is important to emphasize that since above two integrations are around infinity, in general we can not change the ordering (see the discussion in the appendix A), i.e., $B^{\langle 12| n]} \neq B^{\langle 21| n]}$. Nevertheless, we can still change the order of $w$ and $z_{i}$ integration, and we find

$$
-\operatorname{Res}\left(\frac{B^{\langle 12| n]}}{w}\right)_{w_{\mathcal{I}}}=A_{L}\left(p_{3}-w_{\mathcal{I}} q_{3}, \mathcal{I},-P^{h}\right) \frac{1}{\left(p_{3}+P_{\mathcal{I}}\right)^{2}} B^{\langle 12| n]}\left(P^{-h}, \overline{\mathcal{I}}, p_{n}+w_{\mathcal{I}} q_{3}\right), \quad 1,2 \notin \mathcal{I}
$$

Before ending this section, let us give some remarks. For the application of above result, it seems crucial that there is a choice such that after finite steps, we should have $B^{\langle 1 \cdots k| n]}=0$. In later part of the paper, we will discuss several theories in which such a choice always exists. But there are theories in which the boundary contributions do not vanish after exploiting all shifts. If we define the recursion part of the $i$-th deformation as $A^{\langle 1 \cdots i| n]}$, then we have

$$
A=A^{\langle 1| n]}+A^{\langle 12| n]}+\cdots+A^{\langle 1 \cdots n-2| n]}+B^{\langle 1 \cdots n-2| n]} .
$$

In order for our algorithm to be complete, an efficient method to determine the last boundary contribution, $B^{\langle 1 \cdots n-2| n]}$, is desirable. At the same time, it is equally important to explore whether the later terms in (2.16) are suppressed(for example by some large energy scale). In this case one can use the first several terms as a good approximation of the complete amplitude.

\section{Scalar theory}

Starting from this section, we will demonstrate our algorithm by several examples. The first simple example is the real scalar theory with $\phi^{m}$ interaction term, i.e., the Lagrangian is given by

$$
L=-\frac{1}{2} \partial_{\mu} \phi^{I} \partial^{\mu} \phi^{I}+\frac{\sigma}{m !} \phi^{m} .
$$

The vertex $\phi^{m}$ will contribute possible boundary terms for $n$-point amplitude when $n \geq m$. For $n=m$, the contribution is just $\sigma$ and we could not detect it using pole. Thus we will consider the case $n>m$. In fact, to get nontrivial Feynman diagrams, we need to have $n=2+(m-2) V$ where $V$ is the number of vertices.

It is easy to see that under the primary deformation $\langle 1| n]$, the boundary contribution comes from Feynman diagrams where $1, n$ attach to same vertex (see figure 2 ). If we define a non-overlapping $(m-2)$-splitting $K$ of the set $\{2,3, \cdots, n-1\}$ as

$$
K=\left\{K_{1}, K_{2} \cdots K_{k}\right\}
$$




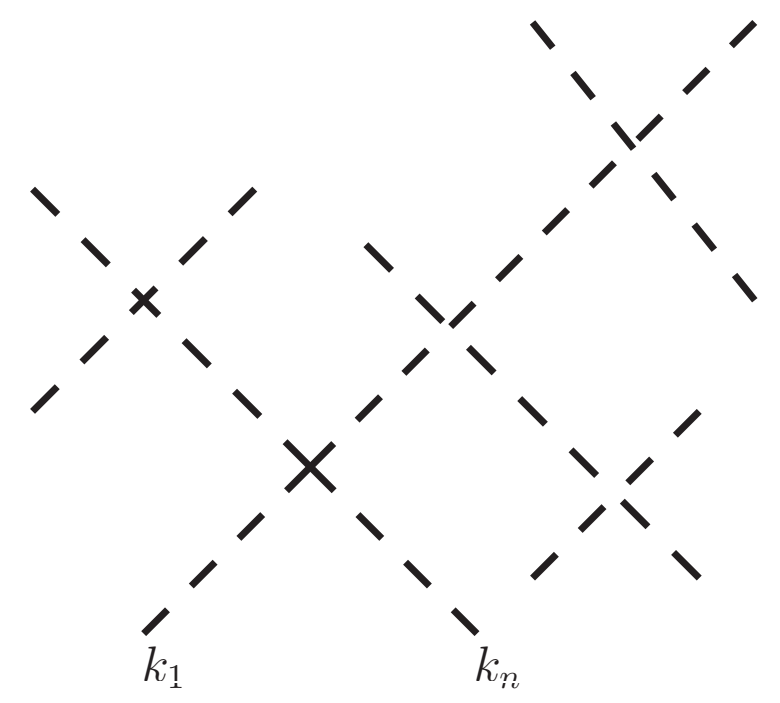

Figure 2. An example of $\mathcal{O}\left(z^{0}\right)$ diagrams under $\left.\langle 1| n\right]$ deformation in $\phi^{4}$ theory.

with each $K_{i}$ having at least one element (the ordering does not matter in the splitting), the boundary contribution is given by

$$
B^{\langle 1| n]}=\sigma \sum_{K \in \Lambda} \frac{1}{p_{K_{1}}^{2} \cdots p_{K_{m-2}}^{2}} \mathcal{A}_{1}\left(K_{1}, P_{1}\right) \cdots \mathcal{A}_{m-2}\left(K_{m-2}, P_{m-2}\right)
$$

where $\Lambda$ is the set of all allowed splitting and $P_{i=1, \ldots, m-2}$ are, in fact, inner particles in figure 2. From (3.3), it is easy to see that since $n>m$, there are at least two vertices. Thus for worst diagrams, where there is only one inner particle connecting to the vertex attached by $1, n$, at most $(m-2)$ new deformations of $\langle i| n]$ type besides $\langle 1| n]$ will be enough to completely determine the boundary contribution $B^{\langle 1| n]}$. For example, for $\phi^{4}$ theory, under the $\langle 1| n](n>4)$, boundary part are given by Feynman diagrams where $1, n$ attach to same vertex. Under the second deformation $\langle 2| n]$, only these Feynman diagrams where 1,2,n attach to same vertex are undetected, but they will be detected by the third deformation, for example, $\langle 3| n]$. Thus by total three steps we can determine the full amplitude.

It is worth to mention that our above discussion of pure scalar theory does not depend on the detail if the theory contains lower point vertex $\phi^{p}$ with $p<m$ in the Lagrangian.

\section{The standard model like theory}

In this section, we discuss standard model like theory, for which the Lagrangian is given by ${ }^{4}$

$$
\begin{aligned}
L & =\operatorname{Tr}\left(L_{0}-\frac{i}{\sqrt{2}}\left(L_{Y}-\bar{L}_{Y}\right)+L_{\phi}\right), \\
L_{0} & =-\frac{1}{4} F_{\mu \nu} F^{\mu \nu}-\frac{1}{2} D_{\mu} \phi^{I} D^{\mu} \phi^{I}+i \bar{\psi}_{A} \bar{\sigma}^{\mu} D_{\mu} \psi^{A},
\end{aligned}
$$

\footnotetext{
${ }^{4}$ In principle, one could add terms $\frac{1}{3} a_{I J K} \phi^{I} \phi^{J} \phi^{K}$ into Lagrangian, but as one can check, our following discussion will not be modified.
} 


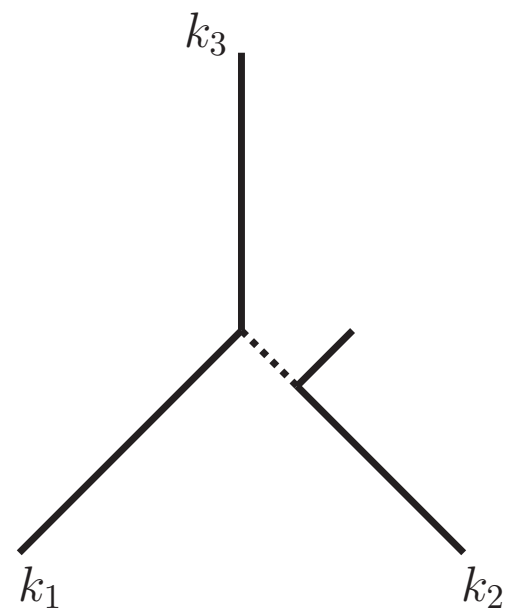

$(a)$

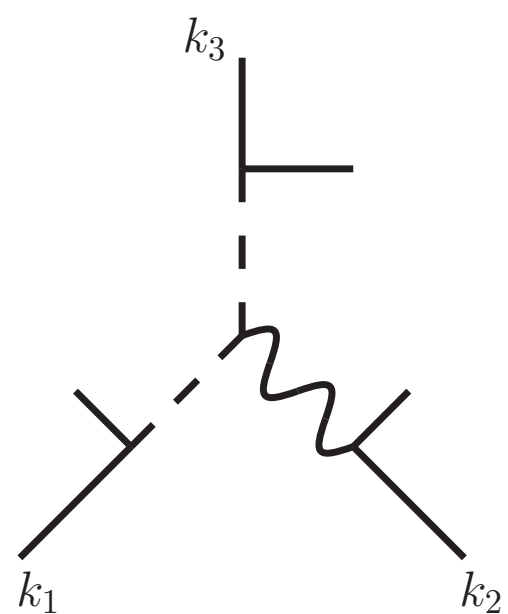

$(b)$

Figure 3. Risager deformation. In case (a), the vetex in the center scales as $z^{0}$, but it is attached to a $\mathcal{O}\left(z^{-1}\right)$ propagator. In case (b), the vertex in the center scales as $z^{1}$, but it is attached to three $\mathcal{O}\left(z^{-1}\right)$ propagators.

$$
\begin{aligned}
L_{Y} & =\lambda_{I A B} \phi^{I} \psi^{A} \psi^{B}, \quad \bar{L}_{Y}=\bar{\lambda}_{I}^{B A} \phi^{I} \bar{\psi}_{A} \bar{\psi}_{B}, \quad \bar{\lambda}_{I}^{A B}=\left(\lambda_{I A B}\right)^{*} \\
L_{\phi} & =\frac{1}{4} a_{I J K L} \phi^{I} \phi^{J} \phi^{K} \phi^{L} .
\end{aligned}
$$

For simplicity, the gauge group is $\mathrm{SU}(N)$ and scalars and fermions are massless. For this theory, we can classify configurations of external particles as following: (a) there is at least one gluon; (b) there is no gluon, but at least four fermions; (c) there are only two fermions and others are scalars; (d) all are scalars.

For the case (a), as has been proved in $[15,16]$, if external particles contain at least one gluon, there is always a good deformation without boundary contribution, so we will not consider case (a) further.

For case (b), there is also a one-step deformation to completely determine amplitude. If there is at least three positive fermions, for example, 1,2, 3 , we can make following Risager deformation [17],

$$
\begin{aligned}
& |1\rangle \rightarrow|1\rangle+z[23]|\eta\rangle, \\
& |2\rangle \rightarrow|2\rangle+z[31]|\eta\rangle, \\
& |3\rangle \rightarrow|3\rangle+z[12]|\eta\rangle .
\end{aligned}
$$

Now we count the power of $z$. First the wave functions of three particles are |1], |2], |3], so they scale as $z^{0}$. Next, each fermionic propagator scales as $z^{0}$ while each bosonic propagator scales as $z^{-1}$. Last, among the vertices, only $A^{3}, A \phi \partial \phi$ contribute $z^{1}$ factors. But since these vertices are attached to at least two ${ }^{5}$ bosonic propagators, thus the number of vertices is always less than the number of bosonic propagator (see, figure 3), thus under the large

\footnotetext{
${ }^{5} \mathrm{~A}$ vertex can be attached to three deformed bosonic propagators, e.g. the vertex in the center of figure $3(\mathrm{~b})$.
} 
$z$ limit, the integrand vanishes. If there are two positive fermion $\psi_{1}, \psi_{2}$ and two negative fermion $\psi_{3}, \psi_{4}$, we can do following deformation,

$$
\begin{aligned}
& |1\rangle \rightarrow|1\rangle+z x_{1}|3\rangle, \\
& |2\rangle \rightarrow|2\rangle+z x_{2}|3\rangle, \\
& \left.\left.\mid 3] \rightarrow \mid 3]-z\left(x_{1} \mid 1\right]+x_{2} \mid 2\right]\right) .
\end{aligned}
$$

Since under this deformation, the wave functions of $\psi_{i}, i=1,2,3$ are not changed, the power counting of $z$ is similar to the case where all $\psi_{i}, i=1,2,3$ are positive. The deformation (4.3) is kindly of the union of two BCFW-deformation using same $z$ variable.

In the next several subsections we will show that for cases (c) and (d), the amplitude can be computed by a (at most) 4 step recursion.

\subsection{The case (c) with only two fermions}

Now we consider $n$-point amplitude with 2 fermions and $(n-2)$ scalars. With out loss of generality we assume the particles 1,2 are scalars while $n$ is the negative fermion, so its wave function is given by $|n\rangle$. We will work in light cone gauge, which is most convenient for the analysis of boundary behavior.

Since we are mainly interested in gauge coupling, we will neglect Yukawa and quartic scalar coupling terms in (4.1) for now. The Light-cone gauge Lagrangian is given by

$$
\begin{aligned}
L_{(2)}= & A^{\bar{h}} \partial^{2} A^{h}-\frac{1}{2} \partial_{\mu} \phi^{I} \partial^{\mu} \phi^{I}+i \bar{\psi}_{A} \bar{\sigma}^{\mu} \partial_{\mu} \psi \\
L_{(3)}= & -2 i g\left(\frac{\partial^{h}}{\partial^{-}} A^{\bar{h}}\right)\left[A^{\bar{h}}, \partial^{-} A^{h}\right]-2 i g\left(\frac{\partial^{\bar{h}}}{\partial^{-}} A^{h}\right)\left[A^{h}, \partial^{-} A^{\bar{h}}\right] \\
& +g A^{h}\left(\mathcal{J}^{\bar{h}}-\frac{\partial^{\bar{h}}}{\partial^{-}} \mathcal{J}^{-}\right)+g A^{\bar{h}}\left(\mathcal{J}^{h}-\frac{\partial^{h}}{\partial^{-}} \mathcal{J}^{-}\right) \\
L_{(4)}= & 2 g^{2} \frac{1}{\partial^{-}}\left[A^{h}, \partial^{-} A^{\bar{h}}\right] \frac{1}{\partial^{-}}\left[A^{\bar{h}}, \partial^{-} A^{h}\right]-\frac{g^{2}}{2}\left(\frac{1}{\partial^{-}} \mathcal{J}^{-}\right)^{2} \\
& +g^{2} \frac{1}{\partial^{-}} \mathcal{J}^{-}\left(\frac{-i}{\partial^{-}}\left[A^{h}, \partial^{-} A^{\bar{h}}\right]-\frac{i}{\partial^{-}}\left[A^{\bar{h}}, \partial^{-} A^{h}\right]\right) \\
\mathcal{J}^{\mu}= & i g\left[\phi^{I}, \partial^{\mu} \phi^{I}\right]-g\left[\bar{\psi}_{A} \bar{\sigma}^{\mu}, \psi^{A}\right]
\end{aligned}
$$

where $A^{+}=\bar{q} \cdot A, A^{-}=q \cdot A, A^{h}=h \cdot A$ and $A^{\bar{h}}=\bar{h} \cdot A$ with the basis $q, \bar{q}, h, \bar{h}$ defined as

$$
q^{\mu}=\frac{1}{2}\left[1\left|\gamma^{\mu}\right| n\right\rangle, \quad \bar{q}^{\mu}=\frac{\left[n\left|\gamma^{\mu}\right| 1\right\rangle}{2 k_{1} \cdot k_{n}}, \quad h^{\mu}=\frac{k_{n}^{\mu}}{k_{1} \cdot k_{n}}, \quad \bar{h}^{\mu}=k_{1}^{\mu}
$$

All inner products of basis vanish except $q \cdot \bar{q}=-1, h \cdot \bar{h}=1$. The advantage of Light-cone gauge Lagrangian (4.4) is that under the deformation $\langle i| n]$, many $z$-factor coming from vertices will be canceled out. For example, for $L_{(4)}$ part, only $\partial^{-}$operator appears, but it is equal to $p^{-}=q \cdot p$, so under the deformation $\left.\langle i| n\right]$, the $z$-dependent part will be $p^{-}(z) \sim q \cdot\left(z q_{i}\right)=0$. In other words, four point vertex in Light-cone gauge will never contribute $z$ factor. Similar observation can be made for $L_{(3)}$ part. Now we will have 


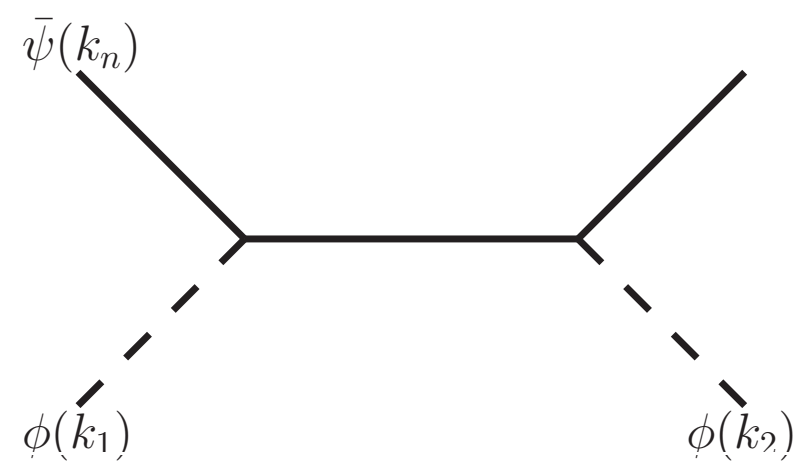

Figure 4. The simpliest diagram in $\left.\left\langle\phi\left(k_{1}\right) \phi\left(k_{2}\right)\right| \bar{\psi}\left(k_{n}\right)\right]$ shift.

$p^{h}=p \cdot h$ and $p^{\bar{h}}=p \cdot \bar{h}$. Under the deformation, we will have $\left(z q_{i}\right) \cdot h=0$ although $\left(z q_{i}\right) \cdot \bar{h} \neq 0$ when $i \neq 1$. In other words, under the $\left.\langle i| n\right]$-deformation, only $A^{h} A^{\bar{h}} A^{\bar{h}}$ vertex, $\phi \phi A^{\bar{h}}$ vertex and $A^{\bar{h}} \psi^{+} \psi^{-}$vertex contribute factor $z$. We will use this important observation to discuss the large $z$ behavior.

Now we consider the boundary contribution under the primary deformation $\langle 1| n]$. First by our above analysis, vertices of $L_{(3)}, L_{(4)}$ will not contribute $z$ factors. The $z$ dependence coming from bosonic and fermionic propagators. Since bosonic propagator is $\frac{1}{z}$ and fermionic propagator is $\frac{1}{z^{0}}$, the worst Feynman diagrams are these without bosonic propagators and scale as $\frac{1}{z^{0}}$. Now we consider the vertex $n$ attached. If 1 is not attached to same vertex, there is one fermionic propagator depending on $z$ connected to $n$. If it is Yukawa coupling we will have $\frac{|P+z q| n\rangle}{(P+z q)^{2}}=\frac{|P| n\rangle}{(P+z q)^{2}}$. If it is gauge coupling we will have $\frac{|P+z q| \gamma^{\mu}|n\rangle}{(P+z q)^{2}}=\frac{|P| \gamma^{\mu}|n\rangle+z|n\rangle q^{\mu}}{(P+z q)^{2}}$ where we have used $\left.\left|q \gamma^{\mu}\right| n\right\rangle=|n\rangle\left[1\left|\gamma^{\mu}\right| n\right\rangle \sim|n\rangle q^{\mu}$. However, under the Light-cone gauge, $z|n\rangle q^{\mu} \cdot A^{\mu}=0$. Thus there is an overall $\frac{1}{z}$ contribution from the vertex $n$ attached and these Feynman diagrams do not give boundary contribution.

By above analysis, we see that boundary contribution comes from these diagrams 1,n attached to same vertex. For these diagrams, we take the second deformation $\langle 2| n]$. Similar analysis as above, only fermionic hard lines (i.e., propagators depending on $z$ ) matter. The simplest diagram of the second shift is shown in figure 4 . Now let us concentrate to the vertex 1, $n$ attached. Using Feynman rule, we have expression like

$$
\frac{\left(k_{1}+k_{n}+z q_{i}\right)|n\rangle}{\left(k_{1}+k_{n}+z q_{i}\right)^{2}}=\mathcal{O}\left(\frac{1}{z}\right)
$$

Thus by two steps, our algorithm can determine whole amplitude in the case (c).

\subsection{The case (d) with only scalars}

Now we consider the large $z$ behavior under the primary deformation $\langle 1| n]$. First there is a special diagram (see figure 5(c)) for which the Light-cone Lagrangian (4.4) is not well defined. It scales as $\mathcal{O}(z)$. For other diagrams, we can use Light-cone Lagrangian (4.4) to analyze. The good point is that all vertices does not give any $z$ contribution. Furthermore, since all external particles are scalars, there is no fermionic propagator. Thus we are left 


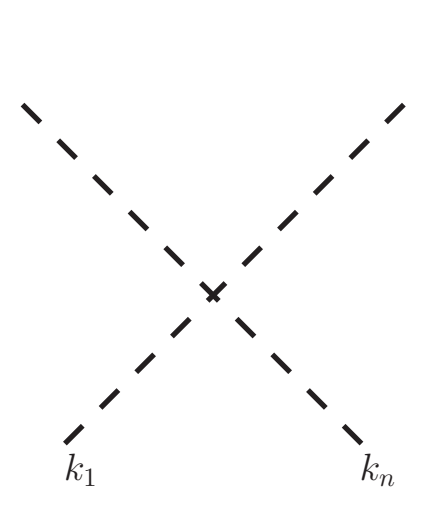

$(a)$

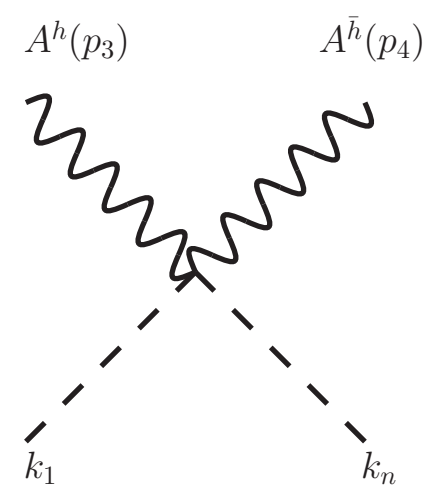

$(b)$

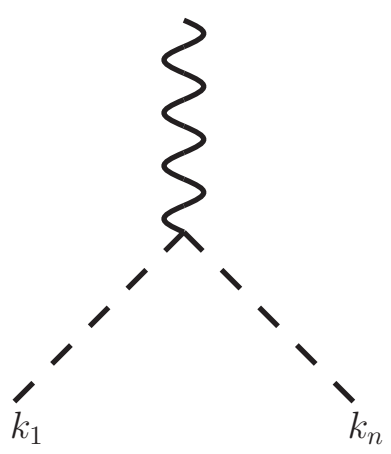

$(c)$

Figure 5. Diagrams contributes to boundary terms in a $\left.\left\langle\phi\left(k_{1}\right)\right| \phi\left(k_{n}\right)\right]$ shift. The third diagram scales as $\mathcal{O}(z)$, while the other two diagrams scale as $\mathcal{O}\left(z^{0}\right)$.

with only bosonic propagators with scale $\frac{1}{z}$. To have boundary contribution, there should be no any propagator depending on $z$, thus we are left with only two types of diagrams (see figure $5(\mathrm{a}),(\mathrm{b})$ ). Now we analyze these three types of diagrams one by one.

Type (a): first let us consider the second deformation $\langle 2| n]$. Under this deformation, only $\partial^{\bar{h}}$ operator contributes $z$ in (4.4). Thus when combining $\frac{1}{z}$ contributions from each bosonic propagator, only type given in figure 6 has $\mathcal{O}(1)$ large $z$ behavior. In particular, the hard line (i.e., the $z q$ floating line) can not have gluon propagator. Otherwise, it will be at least the $\frac{1}{z}$ scaling. For this type of diagrams, the $z$-dependent part can be written down as

$$
\frac{\left(2 k_{2}+p_{1}\right) \cdot \bar{e}\left(p_{1}\right)\left(2 k_{2}+2 p_{1}+p_{2}\right) \cdot \bar{e}\left(p_{2}\right) \cdots\left(2 k_{2}+2 p_{1}+\cdots 2 p_{l-1}+p_{l}\right) \cdot \bar{e}\left(p_{l}\right)}{2^{\frac{l}{2}}\left(k_{2}+p_{1}\right)^{2}\left(k_{2}+p_{1}+p_{2}\right)^{2} \cdots\left(k_{2}+p_{1}+\cdots p_{l}\right)^{2}}
$$

where $k_{2}, k_{n}$ are shifted and $\bar{e}(p)=\bar{h}-\frac{\bar{h} \cdot p}{q_{1} \cdot p}$ (and similarly $e(p)=h-\frac{h \cdot p}{q_{1} \cdot p}$ ). From it, we can read out boundary of the deformation $\langle 2| n]$ as

$$
\frac{\left(q_{2} \cdot k_{1}\right)^{l}}{2^{\frac{l}{2}} q_{2} \cdot p_{1} q_{2} \cdot\left(p_{1}+p_{2}\right)^{2} \cdots q_{2} \cdot\left(p_{1}+\cdots p_{l}\right)^{2}}
$$

multiplying by other factors from remaining part of Feynman diagrams.

For the third deformation $\langle 3| n]$, by similar analysis, especially there is no gluon propagator along the hard line, only the type of diagrams in figure 7 scales as $\mathcal{O}(1)$. It is worth to mention that factor (4.8) is not affected by the deformation $\langle 3| n]$ although other part of Feynman diagrams will be affected in general.

Finally for the fourth deformation $\langle 4| n]$, by similar analysis, especially when the hard line has gluon propagators scaling behavior will be suppressed by an extra $\frac{1}{z}$ factor, we found that no matter how we insert the particle 4 into figure 7 , we will always get at least $\frac{1}{z}$ scaling. Thus by three steps, we can completely determine boundary contributions of figure $5(\mathrm{a})$. 


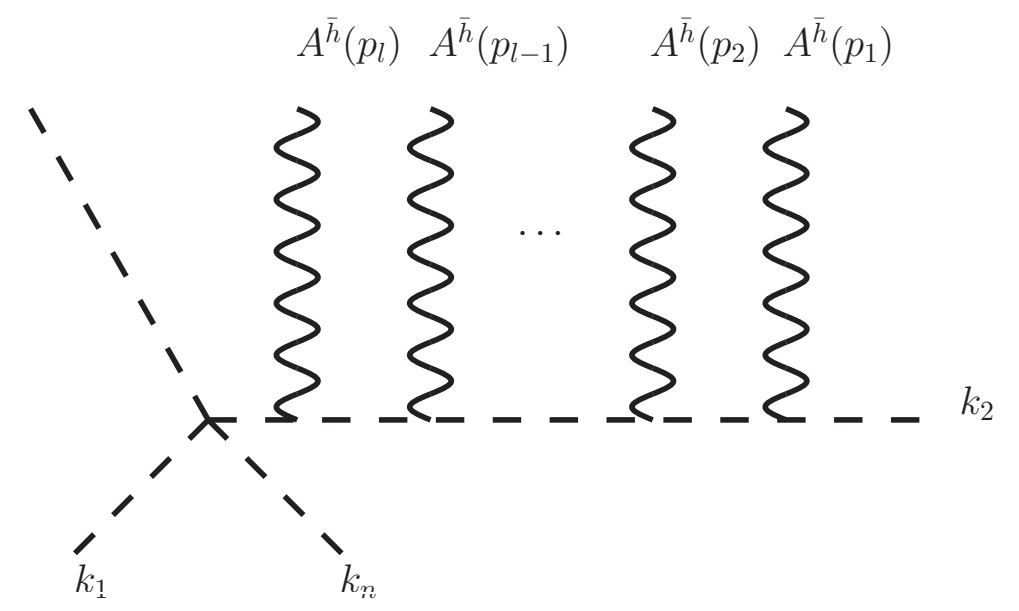

Figure 6. A $\mathcal{O}\left(z^{0}\right)$ diagram in a $\left.\left\langle\phi\left(k_{1}\right) \phi\left(k_{2}\right)\right| \phi\left(k_{n}\right)\right]$ shift.

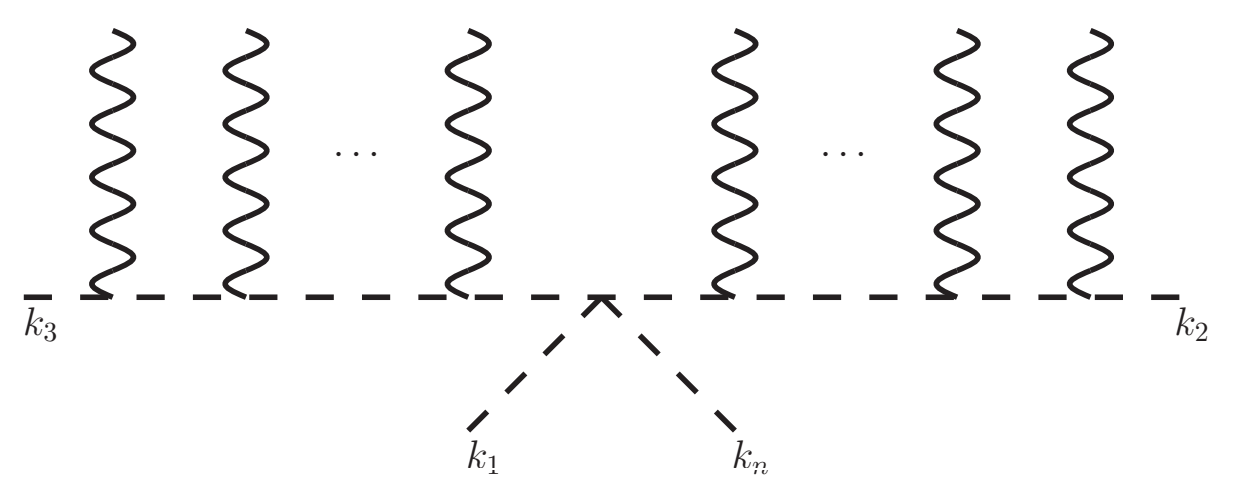

Figure 7. A $\mathcal{O}\left(z^{0}\right)$ diagram in a $\left.\left\langle\phi\left(k_{1}\right) \phi\left(k_{2}\right) \phi\left(k_{3}\right)\right| \phi\left(k_{n}\right)\right]$ shift.

Type (b): now we consider the second deformation $\langle 2| n]$ for the type (b) in figure 5 . Unlike the type (a) where $1, n$ are attached to $\phi^{4}$ vertex, here $1, n$ are attached to $\phi^{2} A^{h} A^{\bar{h}}$ vertex. If the particle 2 is along the line $A^{\bar{h}}$, it can be shown using Lagrangian (4.4) that the large $z$ behavior is $\frac{1}{z}$ at least. But if particle 2 is along the line $A^{h}$, it will contribute to boundary part. After this we will get diagrams like these given in figure 8. Next we consider the third deformation $\langle 3| n]$. There are two cases. For the first case 3 is not directly connected to 2 by scalar line, thus using the same analysis for the type (a), it is $\frac{1}{z}$ behavior at least. For the second case, 3 is directly connected to 2 by scalar line, thus like the figure 7 , it gives nonzero boundary contributions. Finally, like the case (a), the fourth deformation $\langle 4| n]$ will make diagrams in 8 vanishing at large $z$ limit.

Type (c): the type (c) of figure 5 is most complicated one because under our light-cone gauge choice, $p_{1 n} \cdot q=0$, thus we can not impose Light-cone gauge on the gauge field $A$. To solve this problem, we shift momentum basis to

$$
q_{\epsilon}^{\mu}=\frac{1}{2}\left[1\left|\gamma^{\mu}\right| n_{\epsilon}\right\rangle, \quad \bar{q}_{\epsilon}^{\mu}=\frac{\left[n\left|\gamma^{\mu}\right| 1\right\rangle}{2 k_{1} \cdot k_{n \epsilon}}, \quad h_{\epsilon}^{\mu}=\frac{k_{n \epsilon}^{\mu}}{k_{1} \cdot k_{n \epsilon}}, \quad \bar{h}_{\epsilon}^{\mu}=k_{1}
$$




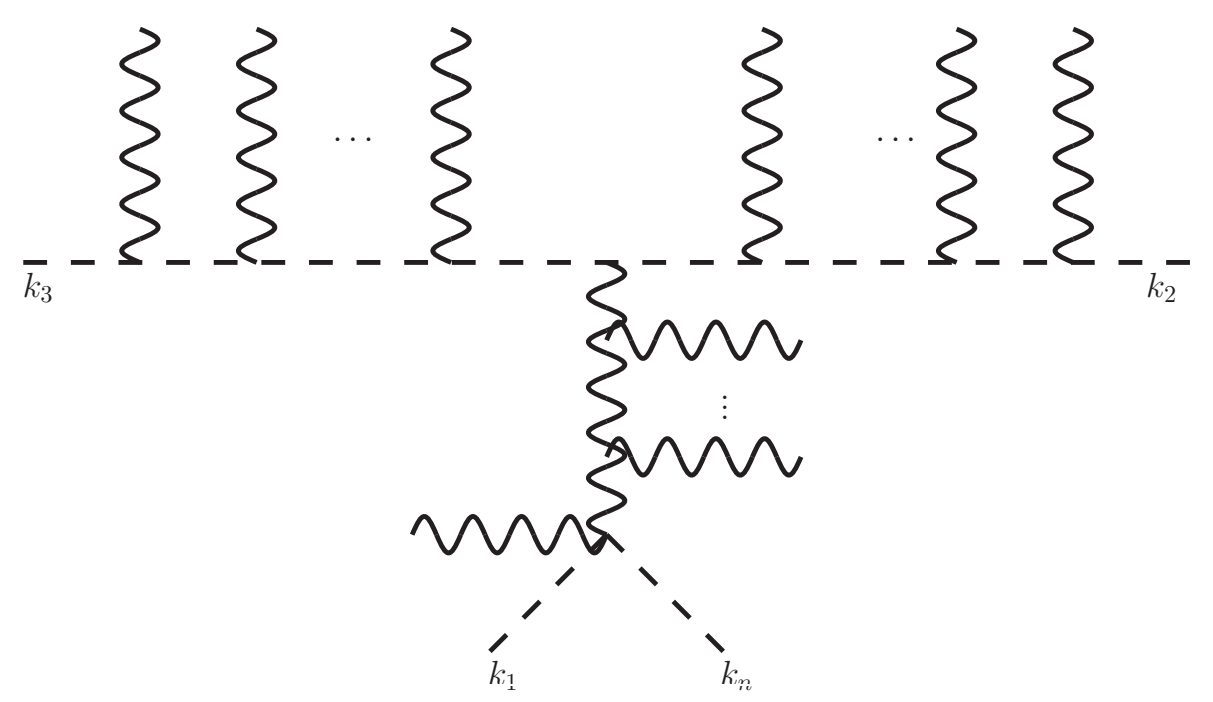

Figure 8. A $\mathcal{O}\left(z^{0}\right)$ diagram in a $\left.\left\langle\phi\left(k_{1}\right) \phi\left(k_{2}\right) \phi\left(k_{3}\right)\right| \phi\left(k_{n}\right)\right]$ shift.

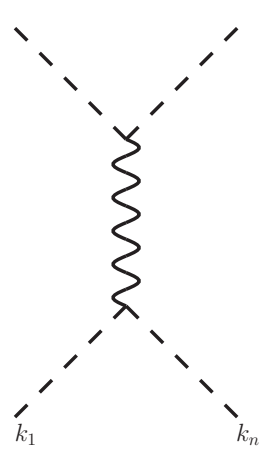

$(c 1)$

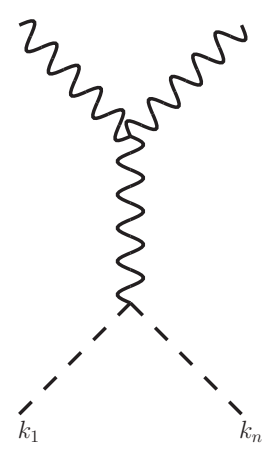

$(c 2)$

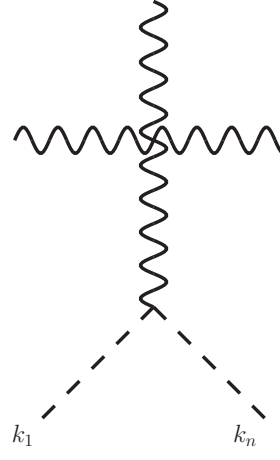

$(c 3)$

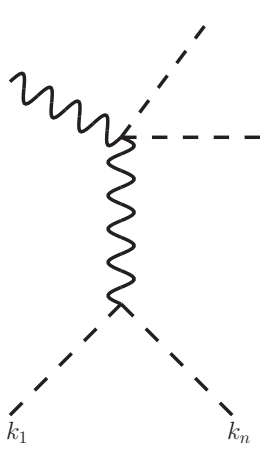

$(c 4)$

Figure 9. Four different diagrams containing the $A \phi \phi$ vertex with $q \cdot P=0$ for the gluon.

where $\left.\left|n_{\epsilon}\right\rangle=\mid n\right]+\epsilon|y\rangle$. Thus when we take $\epsilon \rightarrow 0$ after finishing calculations, we will come back to original light-cone gauge. The type (c) can grow to following four diagrams given in figure 9. Now we discuss these four diagrams one by one.

For diagram (c1), using Feynman rules given in (4.4), it is easy to find

$$
\begin{aligned}
& \frac{1}{\sqrt{2}}\left(k_{1}-k_{n}\right) \cdot \bar{e}_{\epsilon}=\frac{1}{\sqrt{2}}\left(k_{1}-k_{n}\right) \cdot\left(\bar{h}_{\epsilon}-\frac{\left(k_{1}+k_{n}\right) \cdot \bar{h}_{\epsilon}}{\left(k_{1}+k_{n}\right) \cdot q_{\epsilon}} q_{\epsilon}\right)=0 \\
& \frac{1}{\sqrt{2}}\left(k_{1}-k_{n}\right) \cdot e_{\epsilon}=\frac{1}{\sqrt{2}}\left(k_{1}-k_{n}\right) \cdot\left(h_{\epsilon}-\frac{\left(k_{1}+k_{n}\right) \cdot h_{\epsilon}}{\left(k_{1}+k_{n}\right) \cdot q_{\epsilon}} q_{\epsilon}\right)=\sqrt{2}
\end{aligned}
$$

thus If the bottom vertex is $M H V$, the diagram vanishes. If the bottom vertex is $\overline{M H V}$, the diagram reads

$$
\frac{1}{\left(k_{1}+k_{n}\right)^{2}}\left(p_{5}-p_{6}\right) \cdot \bar{e}_{\epsilon}=\frac{1}{2 k_{1} \cdot k_{n}}\left(p_{5}-p_{6}\right) \cdot\left(\bar{h}_{\epsilon}-\frac{k_{n} \cdot \bar{h}_{\epsilon}}{k_{n} \cdot q_{\epsilon}} q_{\epsilon}\right)
$$


This quantity goes to infinity when $\epsilon \rightarrow 0$. However, for four scalars, term $\left(\frac{1}{\partial^{-}} \mathcal{J}^{-}\right)^{2}$ in the $L_{(4)}$ part of Lagrangian will be singular too. Its contribution is

$$
-\frac{1}{2} \frac{\left(k_{1}-k_{n}\right) \cdot q_{\epsilon}\left(p_{5}-p_{6}\right) \cdot q_{\epsilon}}{\left[\left(k_{1}+k_{n}\right) \cdot q_{\epsilon}\right]^{2}}
$$

When combining these two together, we arrive

$$
\frac{1}{2 k_{1} \cdot k_{n}}\left(p_{5}-p_{6}\right) \cdot\left(\bar{h}_{\epsilon}-\frac{k_{n} \cdot \bar{h}_{\epsilon}}{k_{n} \cdot q_{\epsilon}} q_{\epsilon}\right)-\frac{1}{2} \frac{\left(k_{1}-k_{n}\right) \cdot q_{\epsilon}\left(p_{5}-p_{6}\right) \cdot q_{\epsilon}}{\left[\left(k_{1}+k_{n}\right) \cdot q_{\epsilon}\right]^{2}}=\frac{k_{1} \cdot\left(p_{5}-p_{6}\right)}{2 k_{1} \cdot k_{n}}
$$

where the $\epsilon$ has been canceled out.

It is worth to notice that (4.13) scales as $\mathcal{O}(z)$ for $\langle 1| n]$-deformation, but under $\langle i| n]$ deformation it scales as $\mathcal{O}(1)$ ( notice that $p_{5}$ or $p_{6}$ will be shifted). Thus after two steps of $\langle 1| n]$ and $\langle 2| n]$, we arrive to the similar structure as figure 6 . Thus by same argument, two further deformations will enable us to detect all contributions.

For the diagram (c2), the same bottom vertex must be $\overline{M H V}$ to give nonzero contribution. If the top vertex is $\overline{M H V}$, it reads $\left(\frac{p_{5}^{h}}{p_{5}^{-}}-\frac{p_{6}^{h}}{p_{6}^{-}}\right) p^{-}$, thus $p^{-}=q_{\epsilon} \cdot p$ vanishes when $\epsilon \rightarrow 0$. For the top vertex to be $M H V$, we choose the leg $p_{5}$ to have positive helicity, then the diagram gives (including the contribution of 4-vertex) $-\frac{\sqrt{2} k_{1} \cdot p_{6}}{\left(k_{1}+k_{n}\right)^{2}}+\frac{1}{2 \sqrt{2}}+\mathcal{O}(\epsilon)$. In fact, this calculation shows that the diagram (c2) is similar to the type (b) of figure 5 . Thus by same argument, we need at most four steps of deformations to determine its contributions.

For the last diagrams (c3), (c4), to have nonzero contribution, the bottom vertex must be $\overline{M H V}$, while the top vertex is $z^{0}$ scaling. Thus under the second deformation $\left.\langle 2| n\right]$, whole diagram scales as $\frac{1}{z}$. In other words, by two steps of deformations, we can determine its contributions.

Conclusion: after above careful analysis, we can see that for all external particles to be scalars, at most four steps of deformations are enough to completely determine amplitudes by our algorithm.

\section{Examples}

In this section, we will use two examples to demonstrate our method. These two examples correspond to the case (c) and (d) in previous section. We will use $A$ and $B$ to denote color ordered amplitudes and boundary contributions, and $\mathcal{A}$ and $\mathcal{B}$ to denote the complete amplitudes and boundary contributions dressed with color factors.

\subsection{Example I: two fermions with three scalars}

The first example we will consider is $\mathcal{A}\left(\bar{\phi}_{3}\left(k_{1}\right) \phi^{1}\left(k_{2}\right) \bar{\phi}_{1}\left(k_{3}\right) \bar{\psi}_{2}\left(k_{4}\right) \bar{\psi}_{1}\left(k_{5}\right)\right)$ in $\mathcal{N}=4 \mathrm{SYM}$. The two fermions are different flavors and complex scalars are $\phi^{i}=\phi^{i 4}$. In fact, in the language of fermionic coordinator, their types are given by $\eta_{1}^{12} \eta_{2}^{14} \eta_{3}^{23} \eta_{4}^{134} \eta_{5}^{234}$, so $1,2,3,4$ appear three times in the superscript. From the known result of $\mathcal{N}=4 \mathrm{SYM}$ theory, we can read out the expression directly as

$$
\mathcal{A}(12345)=\sum_{\sigma \in S_{3}(\{2,3,4\})} A\left(1 \sigma_{2} \sigma_{3} \sigma_{4} 5\right) F^{a_{1} a_{\sigma_{2}} a_{\sigma_{3}} a_{\sigma_{4}} a_{5}}
$$




$$
A\left(1 \sigma_{2} \sigma_{3} \sigma_{4} 5\right)=\frac{-[12][13][24][35]}{\left[1 \sigma_{2}\right]\left[\sigma_{2} \sigma_{3}\right]\left[\sigma_{3} \sigma_{4}\right]\left[\sigma_{4} 5\right][51]}
$$

where we have defined the color factor

$$
F^{a_{1} a_{2} a_{3} a_{4} a_{5}}=f^{a_{1} a_{2} b} f^{b a_{3} c} f^{c a_{4} a_{5}}
$$

For this example, we need two steps to determine the amplitude according to our discussion in case (c) of section four.

We will start with deformation $\langle 1| 5]$. Under this shift, the recursive part gives

$$
\begin{aligned}
& \mathcal{A}\left(\hat{1}, 3,4, \hat{p}_{25}\right) \frac{1}{p_{25}^{2}} \mathcal{A}\left(-\hat{p}_{25}, 2, \hat{5}\right)+\mathcal{A}\left(\hat{1}, 2,3, \hat{p}_{45}\right) \frac{1}{p_{45}^{2}} \mathcal{A}\left(-\hat{p}_{45}, 4, \hat{5}\right) \\
& =\left(\frac{[23]}{[25][34]} F^{a_{1} a_{3} a_{4} a_{2} a_{5}}-\frac{[13][24]}{[14][25][34]} F^{a_{1} a_{4} a_{3} a_{2} a_{5}}\right)+\left(\frac{1}{[45]} F^{a_{1} a_{3} a_{2} a_{4} a_{5}}-\frac{[13][24]}{[14][23][45]} F^{a_{2} a_{3} a_{1} a_{4} a_{5}}\right)
\end{aligned}
$$

Now we calculate the boundary part using the boundary recursion relation with deformation $\langle 2| 5]$. Naively, there will be following splitting diagrams: (1) $\mathcal{A}_{4}(2,3,4, P) \mathcal{B}_{3}^{\langle 1| 5]}(-P, 1,5)$, (2) $\mathcal{A}_{3}(2,3, P) \mathcal{B}_{4}^{\langle 1| 5]}(-P, 1,5,4)$ and $(3) \mathcal{A}_{3}(2,4, P) \mathcal{B}_{4}^{\langle 1| 5]}(-P, 1,5,3)$. Among these three cases, only case (1) gives nonzero contribution. The reason is that the sum $k$ of negative helicity should be four (i.e., we should have $\eta^{A}$ with $A=1,2,3,4$ appear four times), thus since one side is four-point amplitude with $k=2$, another side of three-point amplitude must have $k=2$. This can not be true for $\mathcal{A}_{3}(2,4, P)$ with $\eta_{2}^{14} \eta_{3}^{134} \eta_{P}^{i}$ or $\eta_{2}^{14} \eta_{3}^{134} \eta_{P}^{i j k}$ no matter how we choose $i \neq j \neq k$ from $\{1,2,3,4\}$. For $\mathcal{A}_{3}(2,3, P)$, we need to choose $\eta_{2}^{14} \eta_{3}^{23} \eta_{P}^{1234}$, but since now $\mathcal{A}_{3}$ is MHV amplitude and the deformation of $\lambda_{2}$ makes $\hat{\lambda}_{2} \sim$ $\lambda_{3} \sim \widehat{\lambda}_{P}$, we get zero.

For the remaining case (1), we calculate as following. First from

$$
\mathcal{A}\left(\bar{\psi}_{2}\left(k_{6}\right), \bar{\phi}_{3}\left(k_{1}\right), \bar{\psi}_{1}\left(k_{5}\right)\right)=-\langle 56\rangle f^{a_{1} a_{5} a_{6}}
$$

we find the boundary part

$$
\mathcal{B}_{3}^{\langle 1| 5]}(-P, 1,5)=-\langle 5 P] f^{a_{P} a_{1} a_{5}}
$$

Putting it back we get

$$
\begin{aligned}
\mathcal{B}^{\langle 1| 5]} & =\mathcal{A}_{4}\left(\hat{2}, 3,4, \hat{p}_{15}\right) \frac{1}{p_{15}^{2}} \mathcal{B}_{3}^{\langle 1| 5]}\left(-\hat{p}_{15}, 1, \hat{5}\right) \\
& =\frac{[12][13]}{[14][15][23]} F^{a_{1} a_{5} a_{4} a_{2} a_{3}}-\frac{[13]}{[15][34]} F^{a_{1} a_{5} a_{2} a_{3} a_{4}}
\end{aligned}
$$

It is easy to check that combining (5.6) and (5.3), we do reproduce (5.1).

\subsection{Example II: six scalars}

The theory we are considering is the scalar-Yang-Mills theory

$$
L=\operatorname{Tr}\left(-\frac{1}{4} F_{\mu \nu} F^{\mu \nu}-D_{\mu} \bar{\Phi} D^{\mu} \Phi-\frac{g^{2}}{2}[\Phi, \bar{\Phi}]^{2}\right)
$$


For this theory, a standard method is to consider the color-ordering amplitudes. For sixpoint amplitudes with three + scalars and three - scalars, there are following three primary color ordering amplitudes

$$
\begin{aligned}
A(+++---)= & -\frac{\langle 12\rangle[45][3|4+5| 6\rangle}{\tau_{345}\langle 16\rangle[34][5|3+4| 2\rangle}+\frac{\langle 23\rangle[56][1|5+6| 4\rangle}{\tau_{234}\langle 34\rangle[16][5|3+4| 2\rangle} \\
A(++-+--)= & -\frac{[3|1+2| 4\rangle^{2}\langle 56\rangle[12]}{\tau_{123}[1|2+3| 4\rangle[3|4+5| 6\rangle\langle 45\rangle[23]} \\
& -\frac{[1|2+4| 3\rangle^{2}\langle 24\rangle^{2}[56]}{\tau_{234}[5|3+4| 2\rangle[1|2+3| 4\rangle\langle 23\rangle\langle 34\rangle[16]} \\
& -\frac{[4|3+5| 6\rangle^{2}\langle 12\rangle[35]^{2}}{\tau_{345}[5|3+4| 2\rangle[3|4+5| 6\rangle\langle 16\rangle[34][45]} \\
A(+-+-+-)= & -\frac{[2|4+6| 5\rangle^{2}\langle 46\rangle^{2}[13]^{2}}{\tau_{123}[1|2+3| 4\rangle[3|4+5| 6\rangle\langle 45\rangle\langle 56\rangle[12][23]} \\
& -\frac{[6|1+5| 3\rangle^{2}\langle 24\rangle^{2}[15]^{2}}{\tau_{234}[1|2+3| 4\rangle[5|3+4| 2\rangle\langle 23\rangle\langle 34\rangle[56][16]} \\
& -\frac{[4|3+5| 1\rangle^{2}\langle 26\rangle^{2}[35]^{2}}{\tau_{345}[5|3+4| 2\rangle[3|4+5| 6\rangle\langle 12\rangle\langle 16\rangle[34][45]}
\end{aligned}
$$

We will calculate (5.10) using our algorithm.

We start with deformation $\langle 1| 6]$, the recursive part is given by

$$
\begin{aligned}
\mathcal{A}^{\langle 1| 6]}= & -\frac{\langle 16\rangle[35]^{2}[4|1+6| 2\rangle^{2}}{\tau_{612}\langle 12\rangle[34][45][5|1+6| 2\rangle[3|1+2| 6\rangle}+\frac{[16]\langle 24\rangle^{2}[5|1+6| 3\rangle^{2}}{\tau_{234}[56]\langle 23\rangle\langle 34\rangle[1|2+3| 4\rangle[5|1+6| 2\rangle} \\
& +\frac{[13]^{2}\langle 46\rangle^{2}[1|2+3| 5\rangle^{2}[2|1+3| 6\rangle^{2}}{\tau_{123}[12][23]\langle 45\rangle\langle 56\rangle[1|2+3| 4\rangle[3|1+2| 6\rangle[1|2+3| 6\rangle^{2}}
\end{aligned}
$$

We will use another three deformations to detect the boundary part. In the first step, using

$$
\begin{aligned}
\mathcal{B}^{\langle 1| 6]}\left(g^{-}\left(k_{7}\right), 4,5,6,1\right) & =\frac{[14](-2[15][46]+[14][56])}{[16][17][45][47]} \\
\mathcal{B}^{\langle 1| 6]}\left(\bar{\Phi}\left(k_{7}\right), 5,6,1\right) & =\frac{-\langle 17\rangle\langle 56\rangle-2\langle 15\rangle\langle 67\rangle}{\langle 16\rangle\langle 57\rangle} \\
\mathcal{B}^{\langle 1| 6]}\left(g^{-}\left(k_{7}\right), 6,1\right) & =\frac{\langle 17\rangle\langle 67\rangle}{\langle 16\rangle}
\end{aligned}
$$

we find

$$
\begin{aligned}
\mathcal{A}^{\langle 12| 6]}= & A\left(\hat{2}, 3,-\hat{p}_{23}\right) \frac{1}{p_{23}^{2}} \mathcal{B}^{\langle 1| 6]}\left(\hat{p}_{23}, 4,5, \hat{6}, 1\right)+A\left(\hat{2}, 3,4,-\hat{p}_{234}\right) \frac{1}{p_{234}^{2}} \mathcal{B}^{\langle 1| 6]}\left(\hat{p}_{234}, 5, \hat{6}, 1\right) \\
& +A\left(\hat{2}, 3,4,5, \hat{p}_{16}\right) \frac{1}{p_{16}^{2}} \mathcal{B}^{\langle 1| 6]}\left(-\hat{p}_{16}, \hat{6}, 1\right) \\
= & \frac{[14]\langle 26\rangle\langle 36\rangle([14][5|2+6| 3\rangle-2[15][4|2+6| 3\rangle)}{[45]\langle 23\rangle[1|2+6| 3\rangle[1|2+3| 6\rangle[4|2+3| 6\rangle} \\
& +\frac{[24]^{2}[3|2+4| 6\rangle^{2}(2\langle 15\rangle[2|3+4| 6\rangle+\langle 56\rangle[2|3+4| 1\rangle)}{\tau_{234}[23][34]\langle 16\rangle[2|3+4| 5\rangle[2|3+4| 6\rangle[4|2+3| 6\rangle}
\end{aligned}
$$




$$
+\frac{[26]\langle 35\rangle^{2}[1|2+6| 4\rangle^{2}}{\tau_{345}[16]\langle 34\rangle\langle 45\rangle[2|3+4| 5\rangle[1|2+6| 3\rangle}
$$

In the second step, using

$$
\mathcal{B}^{\langle 12| 6]}\left(g^{-}\left(k_{7}\right), 5,6,1,2\right)=\frac{[25]}{[27][57]}, \quad \mathcal{B}^{\langle 12| 6]}\left(\Phi\left(k_{7}\right), 6,1,2\right)=-1
$$

we find

$$
\begin{aligned}
\mathcal{A}^{\langle 123| 6]} & =A\left(\hat{3}, 4,-\hat{p}_{34}\right) \frac{1}{p_{34}^{2}} \mathcal{B}^{\langle 12| 6]}\left(\hat{p}_{34}, 5, \hat{6}, 1,2\right)+A\left(\hat{3}, 4,5,-\hat{p}_{345}\right) \frac{1}{p_{345}^{2}} \mathcal{B}^{\langle 12| 6]}\left(\hat{p}_{345}, \hat{6}, 1,2\right) \\
& =-\frac{[25]\langle 36\rangle\langle 46\rangle}{\langle 34\rangle[2|3+4| 6\rangle[5|3+4| 6\rangle}+\frac{[35]^{2}[4|3+5| 6\rangle^{2}}{\tau_{345}[34][45][3|4+5| 6\rangle[5|3+4| 6\rangle}
\end{aligned}
$$

In the third step with deformation, using

$$
\mathcal{B}^{\langle 123| 6]}\left(g^{-}\left(k_{7}\right), 6,1,2,3\right)=\frac{[13]^{2}[27]}{[23][37][17]^{2}}
$$

we find

$$
\begin{aligned}
\mathcal{A}^{\langle 1234| 6]} & =A\left(\hat{4}, 5,-\hat{p}_{45}\right) \frac{1}{p_{45}^{2}} \mathcal{B}^{\langle 123| 6]}\left(\hat{p}_{45}, \hat{6}, 1,2,3\right) \\
& =\frac{[13]^{2}[46]\langle 56\rangle[2|4+5| 6\rangle}{[23]\langle 45\rangle[3|4+5| 6\rangle[1|4+5| 6\rangle^{2}}
\end{aligned}
$$

Numerical checking shows $\mathcal{A}=\mathcal{A}^{\langle 1| 6]}+\mathcal{A}^{\langle 12| 6]}+\mathcal{A}^{\langle 123| 6]}+\mathcal{A}^{\langle 1234| 6]}$, although it is a little bit complicated to show it analytically.

\section{Discussions}

In this paper we showed that boundary contributions satisfy similar recursion relations as scattering amplitudes, and presented a new algorithm to compute boundary contributions. We analyzed large $z$ scaling of amplitudes and boundary contributions in standard model like theories via light cone gauge, and gave two explicit calculations.

It is worth noting that although we only discussed on-shell amplitudes, our method can be applied to amplitudes with off shell currents. The recursion relations for amplitudes with off shell currents was discussed in [10], and one complication there was besides physical states $g^{ \pm}$, longitudinal and time-like states also contributes. Fortunately, only physical states contributes in light cone gauge.

In section 2, we mentioned in general two deformations do not commute. It would be interesting to investigate what is the commutator of two deformations. And it might help us to determine the best choice of deformations.

In general, with more derivatives in vertices the large $z$ scaling of amplitudes get worse. Thus for these theories the boundary might not vanish when all possible deformations of the type $\langle i| n]$ are exploited. It would be interesting to decide under which conditions, our new recursion algorithm ends in finite steps. One particular theory is the one with matters 
coupling to gravity. It would be nice if we can give a similar analysis for the theory, like the example (i.e., the standard model like theory) studied in the paper.

Last, let us point out that boundary contributions serves as a bridge between on-shell and off-shell quantities. On one hand, boundary contributions stem from on shell scattering amplitude, and can be computed using on-shell methods. On the other hand, in many cases Feynman diagrams contributing to boundary part $B^{\langle 1| n]}$ have the topology of the vertex $V(1, n, P)$ connected to off-shell current $J(23 \cdots n-1, P)$ (where $P$ is the propagator). So using boundary contributions we can compute off-shell quantities like correlations function effectively.

\section{Acknowledgments}

We thank Radu Roiban, Chenkai Qiao, Junjie Rao and Kang Zhou for helpful discussions. We would also like to thank Rijun Huang, Congkao Wen for pointing out typos in a previous version. This work is supported by Qiu-Shi funding and Chinese NSF funding under contracts No.11031005, No.11135006, No.11125523 and No.10875103, and National Basic Research Program of China (2010CB833000).

\section{A Ordering of integration and residues of multiple variables}

In this part, we discuss some aspects of integration of multiple variables related to our study.

First we recall the Fubini-Tonelli theorem (often just called Fubini's theorem) which states that if $X$ and $Y$ are $\sigma$-finite measure spaces, and if $f$ is a measurable function such that any one of the three integrals

$$
(I)=\int_{X}\left(\int_{Y}|f(x, y)| d y\right) d x, \quad(I I)=\int_{Y}\left(\int_{X}|f(x, y)| d x\right) d y, \quad(I I I)=\int_{X \times Y}|f(x, y)| d(x, y)
$$

is finite, then

$$
\int_{X}\left(\int_{Y} f(x, y) d y\right) d x=\int_{Y}\left(\int_{X} f(x, y) d x\right) d y=\int_{X \times Y} f(x, y) d(x, y)
$$

In other words, the ordering of two integrations can be exchanged. This theorem is very important for our derivation of recursion relation of boundary contributions.

Having known when the ordering of integrations can be exchanged, we present a counter example related to our discussion in the paper. The example is following two integrations

$$
I_{1}=\oint_{z_{1}=0} \frac{d z_{1}}{z_{1}} \oint_{z_{2}=0} \frac{d z_{2}}{z_{2}} \frac{z_{1}+a z_{2}}{z_{1}+b z_{2}}, \quad I_{2}=\oint_{z_{2}=0} \frac{d z_{2}}{z_{2}} \oint_{z_{1}=0} \frac{d z_{1}}{z_{1}} \frac{z_{1}+a z_{2}}{z_{1}+b z_{2}}
$$

It is easy to see, depending on the value of $z_{1}$ we have

$$
I_{12}\left(z_{1}\right) \equiv \oint_{z_{2}=0} \frac{d z_{2}}{z_{2}} \frac{z_{1}+a z_{2}}{z_{1}+b z_{2}}= \begin{cases}1, & z_{1} \neq 0 \\ \frac{a}{b}, & z_{1}=0\end{cases}
$$


thus we have $I_{1}=\oint_{z_{1}=0} \frac{d z_{1}}{z_{1}} I_{12}\left(z_{1}\right)=1$ since no matter how small is the circle around $z_{1}=0$, as long as $z_{1} \neq 0, I_{12}\left(z_{1} \neq 0\right)=1$. Similarly $I_{2}=\oint_{z_{2}=0} \frac{d z_{2}}{z_{2}} I_{21}\left(z_{2}\right)=\oint_{z_{2}=0} \frac{d z_{2}}{z_{2}} \frac{a}{b}=\frac{a}{b}$. Thus we see that $I_{1} \neq I_{2}$, i.e., the ordering of contour integrations can not be exchanged.

The reason of non-commutativity of (A.3) is not exactly the one mentioned in the Fubini-Tonelli theorem. When we parameterize $z_{1}=R_{1} e^{i \theta_{1}}$ and $z_{2}=R_{2} e^{i \theta_{2}}$ with small radius $R_{1}, R_{2}$ for $I_{1}$, we need to impose condition $R_{2}<R_{1}$ to make sure when we evaluate $z_{2}$-integration, only $z_{2}=0$ pole is inside the circle, i.e., it will not contain pole from factor $z_{1}+b z_{2}$. Similarly, for $I_{2}$ we need to have $R_{2}>R_{1}$. Thus when we exchange the ordering, integral regions are, in fact, different.

Example (A.3) is, in fact, one example of residues of multi-variable studied in [18]. Naively exchanging the ordering of integral variables, the residue can be different only up to a sign. However, as pointed out in [19], when the integration is degenerated, we must be careful. It is easy to see that our example belongs to this special case. If we make following transformation of variables $f_{1}=z_{1}\left(z_{1}+b z_{2}\right)$ and $f_{2}=z_{2}$, the Jacobi $\frac{\partial\left(f_{1}, f_{2}\right)}{\partial\left(z_{1}, z_{2}\right)}=2 z_{1}+b z_{1}$ which is zero when $z_{1}=z_{2}=0$.

Now we consider our example using the method given in [19]. There are three factors $z_{1}, z_{2}, z_{1}+b z_{2}$ in denominator, thus there are several possible combinations. For the first combination, we define $f_{1}=z_{1}\left(z_{1}+b z_{2}\right)$ and $f_{2}=z_{2}$, thus using the algorithm in [19] we find $h_{1}=z_{1}^{2}, h_{2}=z_{2}$ and the transformation matrix is given by

$$
\left(\begin{array}{l}
h_{1} \\
h_{2}
\end{array}\right)=\left(\begin{array}{cc}
1 & -b z_{1} \\
0 & 1
\end{array}\right)\left(\begin{array}{l}
f_{1} \\
f_{2}
\end{array}\right) \equiv A\left(\begin{array}{l}
f_{1} \\
f_{2}
\end{array}\right)
$$

Now the integration becomes

$$
\oint d z_{1} d z_{2} \frac{P\left(z_{1}, z_{2}\right)}{f_{1} f_{2}}=\oint \frac{d z_{1}}{h_{1}} \oint \frac{d z_{2}}{h_{2}} P\left(z_{1}, z_{2}\right) \operatorname{det}(A),
$$

Putting $P\left(z_{1}, z_{2}\right)=z_{1}+a z_{2}$ back we get

$$
\oint \frac{d z_{1}}{z_{1}^{2}} \oint \frac{d z_{2}}{z_{2}}\left(z_{1}+a z_{2}\right)=\oint \frac{d z_{1} z_{1}}{z_{1}^{2}} \oint \frac{d z_{2}}{z_{2}}+\oint \frac{d z_{1}}{z_{1}^{2}} \oint \frac{d z_{2} a z_{2}}{z_{2}}=1
$$

For the second combination $\widetilde{f}_{1}=z_{1}$ and $\widetilde{f}_{2}=z_{2}\left(z_{1}+b z_{2}\right)$, we can find $\widetilde{h}_{1}=z_{1}, \widetilde{h}_{2}=z_{2}^{2}$ and the transformation matrix is given by

$$
\left(\begin{array}{l}
\widetilde{h}_{1} \\
\widetilde{h}_{2}
\end{array}\right)=\left(\begin{array}{cc}
1 & 0 \\
\frac{-z_{2}}{b} & \frac{1}{b}
\end{array}\right)\left(\begin{array}{c}
\widetilde{f}_{1} \\
\widetilde{f}_{2}
\end{array}\right)
$$

thus the integration becomes

$$
\oint \frac{d z_{1}}{z_{1}} \oint \frac{d z_{2}}{z_{2}^{2}} \frac{\left(z_{1}+a z_{2}\right)}{b}=\oint \frac{d z_{1}}{z_{1}} \frac{z_{1}}{b} \oint \frac{d z_{2}}{z_{2}^{2}}+\oint \frac{d z_{1}}{z_{1}} \oint \frac{d z_{2}}{z_{2}^{2}} \frac{a z_{2}}{b}=\frac{a}{b}
$$

Finally for the third combination $\widehat{f}_{1}=z_{1} z_{2}, \widehat{f}_{2}=z_{1}+b z_{2}$, we find $\widehat{h}_{1}=z_{1}^{2}, \widehat{h}_{2}=z_{2}^{2}$, thus

$$
\left(\begin{array}{l}
\widehat{h}_{1} \\
\widehat{h}_{2}
\end{array}\right)=\left(\begin{array}{cc}
-b & z_{1} \\
\frac{-1}{b} & \frac{z_{2}}{b}
\end{array}\right)\left(\begin{array}{c}
\widehat{f}_{1} \\
\widehat{f}_{2}
\end{array}\right)
$$


So the integration becomes

$$
\oint \frac{d z_{1}}{z_{1}^{2}} \oint \frac{d z_{2}}{z_{2}^{2}}\left(z_{1}+a z_{2}\right) z_{2}=\oint \frac{d z_{1} z_{1}}{z_{1}^{2}} \oint \frac{d z_{2} z_{2}}{z_{2}^{2}}+\oint \frac{d z_{1}}{z_{1}^{2}} \oint \frac{d z_{2} a z_{2}^{2}}{z_{2}^{2}}=1
$$

From above calculations, we see that different orderings of integrations in (A.3) correspond to different combinations from the point of view of residue of multi-variables.

\section{B Other deformations for boundary}

In the section two, we have written down recursion relation for boundary contributions under the primary deformation $\langle 1| n]$ using second deformation $\langle 2| n]$ ( or further deformations of the type $\langle i| n]$ ). In this appendix, we discuss possible recursion relation using other types of deformations. Before doing so, let us fix the notation that under the primary deformation $\underline{0} \equiv\langle 1| n]$, the amplitude can be written as

$$
A=\mathcal{A}^{\underline{0}}+\mathcal{B}^{\underline{0}}
$$

where $\mathcal{R}^{\underline{0}}$ is the recursive part and $\mathcal{B}^{0}$ is the boundary part we are trying to determine. We will consider two kinds of other deformations. The first one is another BCFW-deformation, for example, $\underline{1} \equiv\langle 2| 3]$. The second one is the Risager's deformation [17] defined by

$$
[i j k \mid \eta] \equiv\left\{\begin{array}{l}
\mid i(z)]=\mid i]-z\langle j \mid k\rangle \eta \\
\mid j(z)]=\mid j]-z\langle k \mid i\rangle \eta \\
\mid k(z)]=\mid k]-z\langle i \mid j\rangle \eta
\end{array}\right.
$$

or

$$
\langle i j k \mid \eta\rangle \equiv\left\{\begin{array}{l}
|i(z)\rangle=|i\rangle-z[j \mid k] \eta \\
|j(z)\rangle=|j\rangle-z[k \mid i] \eta \\
|k(z)\rangle=|k\rangle-z[i \mid j] \eta
\end{array}\right.
$$

Now we discuss them one by one.

\section{B.1 Using Risager's deformation}

Since from (2.6), spurious poles after the primary deformation $\langle 1| n]$ are the type of $\langle n|P| 1]$ with $p_{1}, p_{n} \notin P$, we would like to take following two kinds of deformations $\left[i j k \mid \widetilde{\lambda}_{1}\right]$ (so $i, j, k \neq 1$ ) and $\left\langle i j k \mid \lambda_{n}\right\rangle$ (so $i, j, k \neq n$ ), thus spurious poles are not deformed. To make our discussion more explicitly we will consider the deformation $\underline{1} \equiv\left\langle 234 \mid \lambda_{n}\right\rangle$, thus following propagators $\left(p_{i}+P_{J}\right)^{2},\left(p_{i}+p_{j}+P_{J}\right)^{2}$ with $i \neq j=2,3,4$ and $J \subset\{5,6, \ldots, n-1\}^{6}$ will provide poles under the 1 -deformation. The locations of these poles are

$$
z_{i, J}=\frac{\left(p_{i}+P_{J}\right)^{2}}{\left\langle n\left|p_{i}+P_{J}\right| i\right][j \mid k]}, \quad z_{i j, J}=-\frac{\left(p_{i}+p_{j}+P_{J}\right)^{2}}{\left\langle n\left|p_{i}+p_{j}+P_{J}\right| k\right][i \mid j]}
$$

\footnotetext{
${ }^{6}$ We will also use the notation $\bar{J}$ which means that $J \bigcup \bar{J}=\{5,6, \ldots, n-1\}$.
} 
with $\{i, j, k\}$ to be the cyclic ordering of $\{2,3,4\}$. Using the contour integration $\oint_{\infty} \frac{d z}{z} \mathcal{B}^{0}(z)$, we can derive the recursion relation for boundary part $\mathcal{B}^{0}$ as following

$$
\begin{aligned}
\mathcal{B}^{\underline{0}}= & \mathcal{B}^{\underline{01}}+\sum_{z_{i, J}} \frac{A_{L}\left(\widehat{\lambda}_{i}\left(z_{i, J}\right), P_{J}, \widehat{P}\left(z_{i, J}\right)\right) \mathcal{B}^{\underline{0}}\left(-\widehat{P}\left(z_{i, J}\right), \widehat{\lambda}_{j}\left(z_{i, J}\right), \widehat{\lambda}_{k}\left(z_{i, J}\right), P_{\bar{J}}, p_{1}, p_{n}\right)}{\left(p_{i}+P_{J}\right)^{2}} \\
& +\sum_{z_{i j, J}} \frac{A_{L}\left(\widehat{\lambda}_{i}\left(z_{i j, J}\right), \widehat{\lambda}_{j}\left(z_{i j, J}\right), P_{J}, \widehat{P}\left(z_{i j, J}\right)\right) \mathcal{B}^{\underline{0}}\left(-\widehat{P}\left(z_{i j, J}\right), \widehat{\lambda}_{k}\left(z_{i j, J}\right), P_{\bar{J}}, p_{1}, p_{n}\right)}{\left(p_{i}+p_{j}+P_{J}\right)^{2}}
\end{aligned}
$$

Since all poles can be proved by same method, we will give the proof for pole $z_{i, J}$ only. The residue of pole $z_{i, J}$ is given by

$$
\oint_{z_{i, J}} \frac{d z}{z} \mathcal{B}^{0}\left(\lambda_{i}-z[j \mid k] \lambda_{n}, \lambda_{j}-z[k \mid i] \lambda_{n}, \lambda_{k}-z[i \mid j] \lambda_{n}\right)
$$

where the contour is a small circle around pole $z_{i, J}$. Now we put the expression of $\mathcal{B}^{0}=$ $\oint_{\infty} \frac{d w}{w} A\left(\lambda_{1}-w \lambda_{n}, \widetilde{\lambda}_{n}+w \widetilde{\lambda}_{1}\right)$ back to get

$$
\begin{aligned}
& \oint_{z_{i, J}} \frac{d z}{z} \oint_{w=\infty} \frac{d w}{w} A_{n}\left(\lambda_{1}-w \lambda_{n}, \lambda_{i}-z[j \mid k] \lambda_{n}, \lambda_{j}-z[k \mid i] \lambda_{n}, \lambda_{k}-z[i \mid j] \lambda_{n}, \widetilde{\lambda}_{n}+w \widetilde{\lambda}_{1}\right) \\
& =\oint_{w=\infty} \frac{d w}{w} \oint_{z_{i, J}} \frac{d z}{z} A_{n}\left(\lambda_{1}-w \lambda_{n}, \lambda_{i}-z[j \mid k] \lambda_{n}, \lambda_{j}-z[k \mid i] \lambda_{n}, \lambda_{k}-z[i \mid j] \lambda_{n}, \widetilde{\lambda}_{n}+w \widetilde{\lambda}_{1}\right) \\
& =-\oint_{w=\infty} \frac{d w}{w} \frac{A_{L}\left(\lambda_{i}-z_{i, J}[j \mid k] \lambda_{n}, P_{J}, \widehat{P}\right) A_{R}\left(-\widehat{P}, \ldots, \lambda_{1}-w \lambda_{n}, \lambda_{j}-z_{i, J}[k \mid i] \lambda_{n}, \lambda_{k}-z_{i, J}[i \mid j] \lambda_{n}, \widetilde{\lambda}_{n}+w \widetilde{\lambda}_{1}\right)}{\left(p_{i}+P_{J}\right)^{2}} \\
& =-\frac{A_{L}\left(\lambda_{i}-z_{i, J}[j \mid k] \lambda_{n}, P_{J}, \widehat{P}\right) \mathcal{B}^{0}\left(-\widehat{P}, \ldots, \lambda_{1}, \lambda_{j}-z_{i, J}[k \mid i] \lambda_{n}, \lambda_{k}-z_{i, J}[i \mid j] \lambda_{n}, \widetilde{\lambda}_{n}\right)}{\left(p_{i}+P_{J}\right)^{2}}
\end{aligned}
$$

where at the second line, we have exchanged the ordering of two contour using the FubiniTonelli theorem while at the fourth line, we have used the fact that in the third line, the variable $w$ appears only on $A_{R}$. Thus we have proved the boundary recursion relation (B.5).

\section{B.2 Using the deformation $\langle 2| 3]$}

First, using (2.5), we can see that possible poles of $\mathcal{B}^{0}$ will be followings

$$
\begin{aligned}
& P_{J}^{2}, \quad\left(p_{2}+P_{J}\right)^{2}, \quad\left(p_{3}+P_{J}\right)^{2}, \quad\left(p_{2}+p_{3}\right)^{2}, \quad\left(p_{2}+p_{3}+P_{J}\right)^{2}, \\
& \left\langle n\left|P_{J}\right| 1\right]^{a}, \quad\left\langle n\left|p_{2}+P_{J}\right| 1\right]^{a}, \quad\left\langle n\left|p_{3}+P_{J}\right| 1\right]^{a}, \quad\left\langle n\left|p_{2}+p_{3}+P_{J}\right| 1\right]^{a}, \quad\left\langle n\left|p_{2}+p_{3}\right| 1\right]^{a}
\end{aligned}
$$

where $J \subset\{4,5, \ldots, n-1\}$. From (B.8), we observe a crucial difference between the deformation of $\langle i| n]$ type and the deformation $\langle 2| 3]$ is that spurious poles $\langle n|P| 1]$ could be detected by the deformation $\langle 2| 3]$. Furthermore, the power of spurious poles could be bigger than one. From (B.8), we find locations of poles are ${ }^{7}$

$$
\begin{aligned}
z_{2, J} & =\frac{\left(p_{2}+P_{J}\right)^{2}}{\left\langle 3\left|P_{J}\right| 2\right]}, & z_{3, J} & =-\frac{\left(p_{3}+P_{J}\right)^{2}}{\left\langle 3\left|P_{J}\right| 2\right]} \\
z_{2, J ; s} & =\frac{\left\langle n\left|p_{2}+P_{J}\right| 1\right]}{\langle n \mid 3\rangle[2 \mid 1]}, & z_{3, J ; s} & =-\frac{\left\langle n\left|p_{3}+P_{J}\right| 1\right]}{\langle n \mid 3\rangle[2 \mid 1]},
\end{aligned}
$$

\footnotetext{
${ }^{7}$ It is worth to notice that for spurious poles, $\left\langle n\left|p_{2}+P_{J}\right| 1\right]$ is same to the one $\left\langle n\left|p_{3}+P_{\bar{J}}\right| 1\right]$ with $\bar{J} \equiv$ $\{4,5, \ldots, n-1\}-J$. Thus when we sum over spurious poles, we should avoid the double counting.
} 
thus we can write down following expression for $\mathcal{B}^{0}$ as

$$
\begin{aligned}
\mathcal{B}^{\underline{0}}= & \mathcal{B}^{\underline{01}}-\sum_{z_{2, J}} \oint_{z=z_{2, J}} \frac{d z}{z} \mathcal{B}^{\underline{0}}\left(\lambda_{2}-z \lambda_{3}, \tilde{\lambda}_{3}+z \widetilde{\lambda}_{2}\right)-\sum_{z_{3, J}} \oint_{z=z_{3, J}} \frac{d z}{z} \mathcal{B}^{\underline{0}}\left(\lambda_{2}-z \lambda_{3}, \tilde{\lambda}_{3}+z \tilde{\lambda}_{2}\right) \\
& -\sum_{z_{2, J ; s}} \oint_{z=z_{2, J ; s}} \frac{d z}{z} \mathcal{B}^{-}\left(\lambda_{2}-z \lambda_{3}, \widetilde{\lambda}_{3}+z \widetilde{\lambda}_{2}\right)-\sum_{z_{3, J ; s}} \oint_{z=z_{3, J ; s}} \frac{d z}{z} \mathcal{B}^{\underline{0}}\left(\lambda_{2}-z \lambda_{3}, \widetilde{\lambda}_{3}+z \widetilde{\lambda}_{2}\right)
\end{aligned}
$$

where $\mathcal{B} \underline{01}=\oint_{z=\infty} \frac{d z}{z} \mathcal{B}^{0}\left(\lambda_{2}-z \lambda_{3}, \widetilde{\lambda}_{3}+z \widetilde{\lambda}_{2}\right)$ is the remaining boundary part.

For the contour integration around the pole $z_{2, J}$, we can evaluate as following

$$
\begin{aligned}
& \oint_{z_{2, J}} \frac{d z}{z} \mathcal{B}^{0}\left(\lambda_{2}-z \lambda_{3}, \widetilde{\lambda}_{3}+z \widetilde{\lambda}_{2}\right) \\
& =\oint_{z_{2, J}} \frac{d z}{z} \oint_{w=\infty} \frac{d w}{w} A_{n}\left(\left\{\lambda_{1}-w \widetilde{\lambda}_{n}, \widetilde{\lambda}_{1}\right\},\left\{\lambda_{2}-z \lambda_{3}, \widetilde{\lambda}_{2}\right\},\left\{\lambda_{3}, \widetilde{\lambda}_{3}+z \widetilde{\lambda}_{2}\right\}, \ldots, p_{n-1},\left\{\lambda_{n}, \widetilde{\lambda}_{n}+w \widetilde{\lambda}_{1}\right\}\right) \\
& =\oint_{w=\infty} \frac{d w}{w} \oint_{z_{2, J}} \frac{d z}{z} A_{n}\left(\left\{\lambda_{1}-w \widetilde{\lambda}_{n}, \widetilde{\lambda}_{1}\right\},\left\{\lambda_{2}-z \lambda_{3}, \widetilde{\lambda}_{2}\right\},\left\{\lambda_{3}, \widetilde{\lambda}_{3}+z \widetilde{\lambda}_{2}\right\} \ldots, p_{n-1},\left\{\lambda_{n}, \widetilde{\lambda}_{n}+w \widetilde{\lambda}_{1}\right\}\right) \\
& =-\oint_{\infty} \frac{d w}{w}\left\{\frac{A_{L}\left(\left\{\lambda_{2}-z_{2, J} \lambda_{3}, \widetilde{\lambda}_{2}\right\}, P_{J}, \widehat{P}^{h}\right) A_{R}\left(-\widehat{P}^{-h}, P_{\bar{J}},\left\{\lambda_{1}-w \widetilde{\lambda}_{n}, \widetilde{\lambda}_{1}\right\},\left\{\lambda_{n}, \widetilde{\lambda}_{n}+w \widetilde{\lambda}_{1}\right\},\left\{\lambda_{3}, \widetilde{\lambda}_{3}+z_{2, J} \widetilde{\lambda}_{2}\right\}\right)}{\left(p_{2}+P_{J}\right)^{2}}\right\} \\
& =-\frac{A_{L}\left(\left\{\lambda_{2}-z_{2, J} \lambda_{3}, \widetilde{\lambda}_{2}\right\}, P_{J}, \widehat{P}^{h}\right) \mathcal{B}^{0}\left(-\widehat{P}^{-h}, P_{\bar{J}},\left\{\lambda_{1}, \widetilde{\lambda}_{1}\right\},\left\{\lambda_{n}, \widetilde{\lambda}_{n}\right\},\left\{\lambda_{3}, \widetilde{\lambda}_{3}+z_{2, J} \widetilde{\lambda}_{2}\right\}\right)}{\left(p_{2}+P_{J}\right)^{2}}
\end{aligned}
$$

where in the third line we have exchanged ordering of contour integrations and in the fourth line, we have evaluate $z$-contour integration. Finally since $w$ appears in $A_{R}$ part only, we arrive the fifth line. Similarly we can obtain

$$
\begin{aligned}
& \oint_{z_{3, J}} \frac{d z}{z} \mathcal{B}^{-}\left(\lambda_{2}-z \lambda_{3}, \widetilde{\lambda}_{3}+z \widetilde{\lambda}_{2}\right) \\
& =-\frac{A_{L}\left(\left\{\lambda_{3}, \widetilde{\lambda}_{3}+z_{2, J} \widetilde{\lambda}_{2}\right\}, P_{J}, \widehat{P}^{h}\right) \mathcal{B}^{0}\left(-\widehat{P}^{-h}, P_{\bar{J}},\left\{\lambda_{1}, \widetilde{\lambda}_{1}\right\},\left\{\lambda_{n}, \widetilde{\lambda}_{n}\right\},\left\{\lambda_{2}-z_{2, J} \lambda_{3}, \widetilde{\lambda}_{2}\right\}\right)}{\left(p_{3}+P_{J}\right)^{2}}
\end{aligned}
$$

Now we evaluate the contour integration around the pole $z_{2, J ; s}$ similarly

$$
\begin{aligned}
& \oint_{z_{2, J ; s}} \frac{d z}{z} \mathcal{B}^{0}\left(\lambda_{2}-z \lambda_{3}, \widetilde{\lambda}_{3}+z \widetilde{\lambda}_{2}\right) \\
& =\oint_{z_{2, J ; s}} \frac{d z}{z} \oint_{w=\infty} \frac{d w}{w} A_{n}\left(\left\{\lambda_{1}-w \widetilde{\lambda}_{n}, \widetilde{\lambda}_{1}\right\},\left\{\lambda_{2}-z \lambda_{3}, \widetilde{\lambda}_{2}\right\},\left\{\lambda_{3}, \widetilde{\lambda}_{3}+z \widetilde{\lambda}_{2}\right\}, \ldots, p_{n-1},\left\{\lambda_{n}, \widetilde{\lambda}_{n}+w \widetilde{\lambda}_{1}\right\}\right) \\
& =\oint_{w=\infty} \frac{d w}{w} \oint_{z_{2, J ; s}} \frac{d z}{z} A_{n}\left(\left\{\lambda_{1}-w \widetilde{\lambda}_{n}, \widetilde{\lambda}_{1}\right\},\left\{\lambda_{2}-z \lambda_{3}, \widetilde{\lambda}_{2}\right\},\left\{\lambda_{3}, \widetilde{\lambda}_{3}+z \widetilde{\lambda}_{2}\right\} \ldots, p_{n-1},\left\{\lambda_{n}, \widetilde{\lambda}_{n}+w \widetilde{\lambda}_{1}\right\}\right)
\end{aligned}
$$

Up to this step, there is nothing particular. However, when we try to evaluate $\oint_{z_{2, J ; s}}$ first with $w$ fixed, new phenomenon happens. In fact, the pole $z_{2, J ; s}$ is the large $w$ limit of following two poles $z_{1,2, J}=\frac{\left(p_{1}+p_{2}+P_{J}\right)^{2}-w\left\langle n\left|p_{1}+p_{2}+P_{J}\right| 1\right]}{\left\langle 3\left|p_{1}+p_{2}+P_{J}\right| 2\right]-w\langle n \mid 3\rangle[2 \mid 1]}$ coming from the propagator $\left(p_{1}+p_{2}+P_{J}\right)^{2}$ and $z_{n, 2, J}=\frac{\left(p_{n}+p_{2}+P_{J}\right)^{2}+w\left\langle n\left|p_{n}+p_{2}+P_{J}\right| 1\right]}{\left\langle 3\left|p_{n}+p_{2}+P_{J}\right| 2\right]+w\langle n \mid 3\rangle[2 \mid 1]}$ coming from the propagator $\left(p_{n}+\right.$ $\left.p_{2}+P_{J}\right)^{2}$. Thus (B.13) should become to

$$
\begin{aligned}
& -\oint_{w=\infty} \frac{d w}{w}\left\{\frac{A_{L}\left(\left\{\lambda_{1}-w \widetilde{\lambda}_{n}, \widetilde{\lambda}_{1}\right\},\left\{\lambda_{2}-z_{1,2, J} \lambda_{3}, \widetilde{\lambda}_{2}\right\}, P_{J}, \widehat{P}\right) A_{R}\left(-\widehat{P}, P_{\bar{J}},\left\{\lambda_{3}, \widetilde{\lambda}_{3}+z_{1,2, J} \widetilde{\lambda}_{2}\right\},\left\{\lambda_{n}, \widetilde{\lambda}_{n}+w \widetilde{\lambda}_{1}\right\}\right)}{\left(p_{1}+p_{2}+P_{J}\right)^{2}}\right. \\
& \left.+\frac{A_{L}\left(\left\{\lambda_{n}, \widetilde{\lambda}_{n}+w \widetilde{\lambda}_{1}\right\},\left\{\lambda_{2}-z_{n, 2, J} \lambda_{3}, \widetilde{\lambda}_{2}\right\}, P_{J}, \widehat{P}\right) A_{R}\left(-\widehat{P}, P_{\bar{J}},\left\{\lambda_{3}, \widetilde{\lambda}_{3}+z_{n, 2, J} \widetilde{\lambda}_{2}\right\},\left\{\lambda_{1}-w \widetilde{\lambda}_{n}, \widetilde{\lambda}_{1}\right\}\right)}{\left(p_{n}+p_{2}+P_{J}\right)^{2}}\right\}(\mathrm{B} .14
\end{aligned}
$$


We can not go further from (B.14) since variable $w$ appears in both $A_{L}, A_{R}$. However, although we can not finish the evaluation of $w$-contour integration, each piece in (B.14) depends only on lower point amplitudes. Thus, in a weak sense, it is also a recursion relation.

Similar evaluation around pole $z_{3, J ; s}$ will give

$$
\begin{aligned}
& -\oint_{w=\infty} \frac{d w}{w}\left\{\frac{A_{L}\left(\left\{\lambda_{1}-w \widetilde{\lambda}_{n}, \widetilde{\lambda}_{1}\right\},\left\{\lambda_{3}, \widetilde{\lambda}_{3}+z_{1,3, J} \widetilde{\lambda}_{2}\right\}, P_{J}, \widehat{P}\right) A_{R}\left(-\widehat{P}, P_{\bar{J}},\left\{\lambda_{2}-z_{1,3, J} \lambda_{3}, \widetilde{\lambda}_{2}\right\},\left\{\lambda_{n}, \widetilde{\lambda}_{n}+w \widetilde{\lambda}_{1}\right\}\right)}{\left(p_{1}+p_{3}+P_{J}\right)^{2}}\right. \\
& \left.+\frac{A_{L}\left(\left\{\lambda_{n}, \widetilde{\lambda}_{n}+w \widetilde{\lambda}_{1}\right\},\left\{\lambda_{3}, \widetilde{\lambda}_{3}+z_{n, 3, J} \widetilde{\lambda}_{2}\right\}, P_{J}, \widehat{P}\right) A_{R}\left(-\widehat{P}, P_{\bar{J}},\left\{\lambda_{2}-z_{n, 3, J} \lambda_{3}, \widetilde{\lambda}_{2}\right\},\left\{\lambda_{1}-w \widetilde{\lambda}_{n}, \widetilde{\lambda}_{1}\right\}\right)}{\left(p_{n}+p_{3}+P_{J}\right)^{2}}\right\} \text { (B.15) }
\end{aligned}
$$

with

$$
\begin{aligned}
& z_{1,3, J}=-\frac{\left(p_{1}+p_{3}+P_{J}\right)^{2}-w\left\langle n\left|p_{1}+p_{3}+P_{J}\right| 1\right]}{\left\langle 3\left|p_{1}+p_{3}+P_{J}\right| 2\right]-w\langle n \mid 3\rangle[2 \mid 1]}, \\
& z_{n, 3, J}=-\frac{\left(p_{n}+p_{3}+P_{J}\right)^{2}+w\left\langle n\left|p_{n}+p_{3}+P_{J}\right| 1\right]}{\left\langle 3\left|p_{n}+p_{3}+P_{J}\right| 2\right]+w\langle n \mid 3\rangle[2 \mid 1]}
\end{aligned}
$$

Finally, putting (B.11), (B.12), (B.14), and (B.15) back to (B.10), we get a "weak recursion relation" for $\mathcal{B}^{0}$ using the deformation $\left.\langle 2| 3\right]$.

\section{Light cone propagator}

Although it is not used explicitly, we like to discuss one aspect of light-cone propagator given by

$$
\Pi_{\mu \nu}=\frac{1}{p^{2}}\left(\eta_{\mu \nu}-\frac{q_{\mu} p_{\mu}+p_{\mu} q_{\mu}}{q \cdot p}\right)
$$

Using the basis $q, \bar{q}, h, \bar{h}$ (see (4.5)), it is easy to rewrite it as

$$
\eta_{\mu \nu}-\frac{q_{\mu} p_{\nu}+p_{\mu} q_{\nu}}{q \cdot p}=e_{\mu} \bar{e}_{\nu}+e_{\nu} \bar{e}_{\mu}-\frac{p^{2}}{(q \cdot p)^{2}} q_{\mu} q_{\nu}
$$

where we have defined

$$
e(p)=h-\frac{h \cdot p}{q \cdot p} q, \quad \bar{e}(p)=\bar{h}-\frac{\bar{h} \cdot p}{q \cdot p} q,
$$

which have been used in main text and are proportional to the gluon polarization vectors $\epsilon^{+}(p)$ and $\epsilon^{-}(p)$, respectively. The $p^{2}$ of the term $\frac{p^{2}}{(q \cdot p)^{2}} q_{\mu} q_{\nu}$ will cancel the denominator of $\Pi_{\mu \nu}$, thus this term will give an effective 4-point vertex.

Open Access. This article is distributed under the terms of the Creative Commons Attribution License (CC-BY 4.0), which permits any use, distribution and reproduction in any medium, provided the original author(s) and source are credited.

\section{References}

[1] R. Britto, F. Cachazo and B. Feng, New recursion relations for tree amplitudes of gluons, Nucl. Phys. B 715 (2005) 499 [hep-th/0412308] [INSPIRE]. 
[2] R. Britto, F. Cachazo, B. Feng and E. Witten, Direct proof of tree-level recursion relation in Yang-Mills theory, Phys. Rev. Lett. 94 (2005) 181602 [hep-th/0501052] [INSPIRE].

[3] Z. Bern, L.J. Dixon and D.A. Kosower, On-shell methods in perturbative QCD, Annals Phys. 322 (2007) 1587 [arXiv:0704.2798] [INSPIRE].

[4] B. Feng and M. Luo, An introduction to on-shell recursion relations, Front. Phys. 7 (2012) 533 [arXiv:1111.5759] [INSPIRE].

[5] H. Elvang and Y.-T. Huang, Scattering amplitudes, arXiv:1308.1697 [INSPIRE].

[6] P. Benincasa and F. Cachazo, Consistency conditions on the S-matrix of massless particles, arXiv:0705.4305 [INSPIRE].

[7] R.H. Boels, No triangles on the moduli space of maximally supersymmetric gauge theory, JHEP 05 (2010) 046 [arXiv: 1003.2989] [INSPIRE].

[8] B. Feng, J. Wang, Y. Wang and Z. Zhang, BCFW recursion relation with nonzero boundary contribution, JHEP 01 (2010) 019 [arXiv:0911.0301] [INSPIRE].

[9] B. Feng and C.-Y. Liu, A note on the boundary contribution with bad deformation in gauge theory, JHEP 07 (2010) 093 [arXiv: 1004.1282] [INSPIRE].

[10] B. Feng and Z. Zhang, Boundary contributions using fermion pair deformation, JHEP 12 (2011) 057 [arXiv:1109.1887] [INSPIRE].

[11] P. Benincasa and E. Conde, On the tree-level structure of scattering amplitudes of massless particles, JHEP 11 (2011) 074 [arXiv:1106.0166] [INSPIRE].

[12] P. Benincasa and E. Conde, Exploring the S-matrix of massless particles, Phys. Rev. D 86 (2012) 025007 [arXiv:1108. 3078] [INSPIRE].

[13] B. Feng, Y. Jia, H. Lüo and M. Luo, Roots of amplitudes, arXiv:1111.1547 [INSPIRE].

[14] B. Feng, K. Zhou, C. Qiao and J. Rao, Determination of boundary contributions in recursion relation, JHEP 03 (2015) 023 [arXiv: 1411. 0452] [INSPIRE].

[15] N. Arkani-Hamed and J. Kaplan, On tree amplitudes in gauge theory and gravity, JHEP 04 (2008) 076 [arXiv:0801.2385] [INSPIRE].

[16] C. Cheung, On-shell recursion relations for generic theories, JHEP 03 (2010) 098 [arXiv: 0808.0504] [INSPIRE].

[17] K. Risager, A direct proof of the CSW rules, JHEP 12 (2005) 003 [hep-th/0508206] [INSPIRE].

[18] N. Arkani-Hamed, F. Cachazo, C. Cheung and J. Kaplan, A duality for the S-matrix, JHEP 03 (2010) 020 [arXiv:0907.5418] [INSPIRE].

[19] M. Sogaard and Y. Zhang, Unitarity cuts of integrals with doubled propagators, JHEP 07 (2014) 112 [arXiv: 1403.2463] [INSPIRE]. 